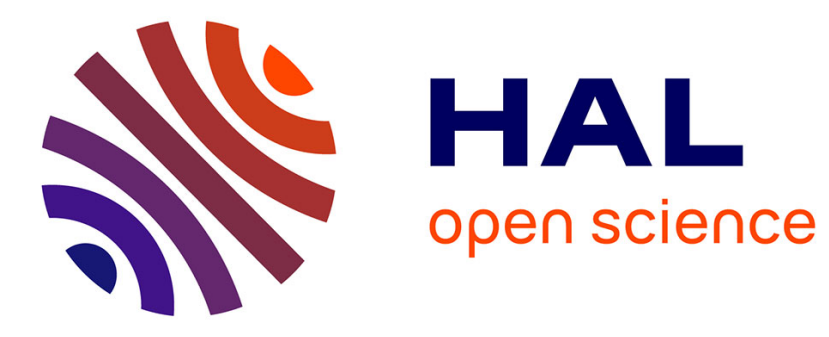

\title{
CD44 regulates epigenetic plasticity by mediating iron endocytosis
}

\author{
Sebastian Müller, Fabien Sindikubwabo, Tatiana Cañeque, Anne Lafon, \\ Antoine Versini, Bérangère Lombard, Damarys Loew, Ting-Di Wu, \\ Christophe Ginestier, Emmanuelle Charafe-Jauffret, et al.
}

\section{To cite this version:}

Sebastian Müller, Fabien Sindikubwabo, Tatiana Cañeque, Anne Lafon, Antoine Versini, et al.. CD44 regulates epigenetic plasticity by mediating iron endocytosis. Nature Chemistry, 2020, 12 (10), pp.929938. 10.1038/s41557-020-0513-5 . hal-03365507

\section{HAL Id: hal-03365507 https://hal.science/hal-03365507}

Submitted on 5 Oct 2021

HAL is a multi-disciplinary open access archive for the deposit and dissemination of scientific research documents, whether they are published or not. The documents may come from teaching and research institutions in France or abroad, or from public or private research centers.
L'archive ouverte pluridisciplinaire HAL, est destinée au dépôt et à la diffusion de documents scientifiques de niveau recherche, publiés ou non, émanant des établissements d'enseignement et de recherche français ou étrangers, des laboratoires publics ou privés. 


\title{
CD44 regulates epigenetic plasticity by mediating iron endocytosis
}

\author{
Sebastian Müller $\mathbb{B}^{1,2,3,11}$, Fabien Sindikubwabo ${ }^{1,2,3,11}$, Tatiana Cañeque ${ }^{1,2,3}$, Anne Lafon ${ }^{1,2,3}$, \\ Antoine Versini ${ }^{1,2,3}$, Bérangère Lombard ${ }^{1,2,4}$, Damarys Loew ${ }^{10,2,4}$, Ting-Di Wu ${ }^{1,2,5}$, \\ Christophe Ginestier ${ }^{6,7}$, Emmanuelle Charafe-Jauffret ${ }^{6,7}$, Adeline Durand ${ }^{1,2,8}$, Céline Vallot ${ }^{1,2,8}$, \\ Sylvain Baulande ${ }^{1,2,9}$, Nicolas Servant ${ }^{1,10}$ and Raphaël Rodriguez $\mathbb{1}^{1,2,3 凶}$
}

CD44 is a transmembrane glycoprotein linked to various biological processes reliant on epigenetic plasticity, which include development, inflammation, immune responses, wound healing and cancer progression. Although it is often referred to as a cell surface marker, the functional regulatory roles of CD44 remain elusive. Here we report the discovery that CD44 mediates the endocytosis of iron-bound hyaluronates in tumorigenic cell lines, primary cancer cells and tumours. This glycan-mediated iron endocytosis mechanism is enhanced during epithelial-mesenchymal transitions, in which iron operates as a metal catalyst to demethylate repressive histone marks that govern the expression of mesenchymal genes. CD44 itself is transcriptionally regulated by nuclear iron through a positive feedback loop, which is in contrast to the negative regulation of the transferrin receptor by excess iron. Finally, we show that epigenetic plasticity can be altered by interfering with iron homeostasis using small molecules. This study reveals an alternative iron-uptake mechanism that prevails in the mesenchymal state of cells, which illuminates a central role of iron as a rate-limiting regulator of epigenetic plasticity.

C D44 is a transmembrane glycoprotein implicated in many physiological and pathological processes, which include development, inflammation, immune responses, wound healing and cancer progression ${ }^{1,2}$. These processes involve cells able to dynamically and reversibly shift between cell states through epithelial-mesenchymal transitions $(\mathrm{EMT})^{3-5}$. For instance, epithelial-mesenchymal plasticity can give rise to cells that exhibit elevated levels of CD44 across lineages in cancers and healthy tissues. Furthermore, cancer cells of a mesenchymal origin, cancer-associated fibroblasts and activated $\mathrm{T}$ cells involved in immune responses can also display CD44 at the plasma membrane. In the context of cancer, diseased mesenchymal cells contribute to drug resistance ${ }^{4}$, and CD44 itself has been shown to confer metastatic potential to these cells $s^{6}$. However, the molecular mechanisms that underlie the functional role of CD44 are not fully understood. $\mathrm{CD} 44$ has been proposed to act as a ligand-binding surface protein that regulates cell adhesion and migration through the extracellular matrix, and as a membrane co-receptor that mediates intracellular signal transduction ${ }^{1}$. Importantly, CD44 interacts with and mediates endocytosis of hyaluronates (Hyal) 7,8 , a class of glycosaminoglycan biopolymers that can promote EMT and tumour development ${ }^{9-11}$.

Hyal exhibit negatively charged carboxylates on every disaccharide unit. This raises the question as to which counterions these biopolymers interact with at a physiological $\mathrm{pH}$ during endocytosis to enable the uptake of Hyal without altering the intracellular ionic balance and membrane potentials. The ability of Hyal to interact with metals, including iron $(\mathrm{Fe})\left(\mathrm{ref} .{ }^{12}\right)$, together with the iron dependency of mesenchymal cancer cells in vivo ${ }^{13-16}$ and their vulnerability to ferroptotic cell death ${ }^{15,17,18}$, prompted us to search for a putative CD44-mediated iron-uptake mechanism. Here we report the discovery of a Hyal-dependent iron endocytosis pathway mediated by CD44, which is upregulated during EMT. We found that an increase of iron uptake is required for the activity of iron-dependent demethylases that regulate the expression of specific genes in the mesenchymal state of cells. This work illuminates a central role of iron in the regulation of epigenetic plasticity functionally linking the membrane glycoprotein CD44 to the nucleus.

\section{Results}

CD44 mediates Hyal-dependent iron endocytosis. We first employed NMR spectroscopy to study the interactions between Hyal and iron. The addition of $\mathrm{Fe}(\mathrm{III})$ to a low-molecular-mass (LMM) Hyal in water led to a line broadening of the proton signals of LMM Hyal, which indicates that this organic substrate interacts with iron at neutral $\mathrm{pH}$ (Fig. 1a). Acidifying the sample to protonate the carboxylates of LMM Hyal and to disrupt organometallic complexes released the metal, and thereby rescued the proton signals of free LMM Hyal (Fig. 1a). These data demonstrate the dynamic and reversible nature of metal coordination by LMM Hyal in near-physiological conditions of solvent, temperature and $\mathrm{pH}$.

To evaluate the potential of high-molecular-mass (HMM) Hyal-based organometallic complexes to be internalized by CD44, we knocked out (ko) CD44 from mesenchymal human mammary HMLER (human mammary large T SV40 epithelial Ras) cells stably expressing high levels of CD44 (HMLER CD44 $\left.4^{\text {high }}\right)^{19}$ (Fig. 1b). For comparison, we independently knocked out TFRC, the gene

\footnotetext{
IInstitut Curie, Paris, France. ${ }^{2}$ PSL Université, Paris, France. ${ }^{3}$ Chemical Biology of Cancer Laboratory, CNRS UMR 3666, INSERM U1143, Paris, France. ${ }^{4}$ Proteomics Mass Spectrometry Laboratory, Paris, France. ${ }^{5}$ Paris-Sud Université, Paris-Saclay Université, CNRS UMR 9187, INSERM U1196, Paris, France. ${ }^{6}$ Centre de Recherche en Cancérologie de Marseille, Institut Paoli-Calmettes, Aix-Marseille Université, Marseille, France. ${ }^{7}$ Epithelial Stem Cells and Cancer Laboratory, CNRS UMR 10668, INSERM U1068, Marseille, France. ${ }^{8}$ Translational Research Department, CNRS UMR 3244, Paris, France. ${ }^{9}$ Institut Curie Genomics of Excellence Platform, Paris, France. ${ }^{10} \mathrm{CBIO}-$ Centre for Computational Biology, INSERM U900, Mines ParisTech, Paris, France. ${ }^{11 T h e s e}$ authors contributed equally: Sebastian Müller, Fabien Sindikubwabo.凶e-mail: raphael.rodriguez@curie.fr
} 
that codes for transferrin receptor protein 1 (TfR1) (Fig. 1b), which mediates endocytosis of transferrin (TF) and plays a central role in the regulation of cellular iron homeostasis.

We then evaluated the capacity of parental cells and ko clones to take up fluorescently labelled HMM Hyal (0.8 MDa). Internalization of Hyal was reduced in CD44 ko and increased in TFRC ko clones compared with that of parental cells (Fig. 1c), which validates the functional role of CD44 in mediating this process. Hyal and TF, which interact with CD44 and TfR1, respectively, predominantly localized in distinct vesicles in the parental cells (Fig. 1d). This allowed for a comparative analysis of metal uptake mediated either by CD44 or TfR1. Thus, we evaluated whether endocytic vesicles contained Fe(II) and Hyal, independent of TF. To this end, we used the Fe(II)-specific turn-on fluorescent probe RhoNox-M, which can detect the presence of Fe(II) in lysosomes ${ }^{20}$. Parental HMLER cells exhibited RhoNox-M- and Hyal-positive vesicles free of TF, as observed by fluorescence microscopy. CD44 ko cells exhibited a lower proportion of RhoNox-M- and Hyal-positive vesicles with an increased number of RhoNox-M- and TF-positive vesicles compared to parental cells, whereas TFRC ko cells showed the opposite trend (Fig. 1d). Consistently, complementing CD44 ko cells with CD44 restored a higher proportion of RhoNox-M- and Hyal-positive vesicles (Fig. 1e). RhoNox-M- and Hyal-positive vesicles were also observed in other cancer cell lines, primary cancer cells, which included circulating tumour cells (CTC) of lung and colon cancers, and in primary human $\mathrm{T}$ cells (Extended Data Fig. 1a,b). Consistently, LNCaP prostate cancer cells, which do not show detectable levels of the CD44 protein, exhibited a low proportion of RhoNox-M- and Hyal-positive vesicles (Extended Data Fig. 1a).

In line with CD44-mediated iron endocytosis, supplementing cells with HMM Hyal (0.6-1 MDa) increased the levels of cellular iron in parental CD $44^{\text {high }}$ and TFRC ko but not in CD44 ko HMLER and MCF7 cells, as defined by the fluorescence of RhoNox-M quantified by flow cytometry (Extended Data Fig. 2a). This was observed across cell types (Extended Data Fig. 2a,b), which indicates this to be a general feature of cells that express the CD44 protein. In CD44-expressing MDA-MB-468 breast cancer cells, iron uptake was more pronounced when the cells were treated with Hyal of higher molecular mass (Extended Data Fig. 2c). Conversely, treating CD44-expressing cells with hyaluronidase or blocking antibodies against CD44 or TfR1 reduced iron uptake (Extended Data Fig. 2d,e). Additionally, supplementing HMLER CD $44^{\text {high }}$ cells with Hyal increased cellular iron, as detected by secondary ion mass spectrometry (SIMS) imaging ${ }^{21}$ and quantified by inductively coupled plasma mass spectrometry (ICP-MS) (Fig. 1f,g). Supplementing HMLER CD44 ${ }^{\text {high }}$ cells with Hyal also led to increased levels of the iron-storage protein ferritin in a CD44-dependent manner (Fig. $1 \mathrm{~h})$, which further demonstrates an increased iron uptake in these conditions.

To evaluate the physiological importance of this pathway, we sorted $\mathrm{CD} 44^{\text {high/low }}$ cell populations from two patient-derived xenografts of breast tumours refractory to conventional chemotherapy, prone to form metastasis and associated with poor outcome. We found that CD44 ${ }^{\text {high }}$ cells contained higher levels of ferritin with reduced levels of TfR1 compared with CD $44^{\text {low }}$ cells (Fig. 1i). Collectively, these data provide evidence that CD44 mediates iron endocytosis in a Hyal-dependent manner.

Prevalence of CD44-mediated iron endocytosis in the mesenchymal state of cells. The mesenchymal state of cells is characterized by elevated levels of CD44 in several lineages ${ }^{22}$. To investigate the cellular changes reliant on CD44 levels, we triggered EMT in triple-negative breast cancer MDA-MB-468, luminal breast cancer MCF7 and HMLER CD44 ${ }^{\text {low }}$ cell lines, primary breast cancer cells and primary lung CTC using epidermal growth factor (EGF), oncostatin M (OSM) or transforming growth factor beta 1 (TGF- $\beta$ ). On treatment, cells shifted from an epithelial to a mesenchymal phenotype according to cell morphology, reduced levels of the epithelial marker E-cadherin and increased levels of CD44 (Fig. 2a). Consistent with EMT induction, levels of the transcription factor snail increased along with those of the mesenchymal markers vimentin and fibronectin (Fig. 2b). Remarkably, CD44 levels increased together with ferritin, whereas levels of TfR1 decreased at a later time point (Fig. 2b). In line with this, CD44 loading at the plasma membrane was enhanced during EMT, which was in contrast to a reduced loading of TfR1 (Fig. 2c and Extended Data Fig. 3a), and the number of RhoNox-M- and Hyal-positive vesicles increased together with cellular iron (Fig. 2d,e and Extended Data Fig. 3b). Importantly, knocking down CD44 antagonized the production of ferritin in cells treated with EGF (Fig. 2f).

Furthermore, RT-qPCR (quantitative PCR with reverse transcription) showed that mRNA levels of CD44 and FTH1 coding for ferritin increased during EMT, whereas that of TFRC decreased (Fig. $2 \mathrm{~g}$ ). These data are in agreement with the well-established negative feedback loop that regulates the biosynthesis of TfR1 at the translational level by excess cytosolic iron ${ }^{23,24}$. They also indicate that, in contrast to TFRC, CD44 is not subjected to this regulatory loop. Together, these results advocate an alternative glycan-mediated iron endocytosis pathway reliant on CD44 that prevails in the mesenchymal state of cells.

EMT is characterized by a redox signature implicating iron. To identify iron-dependent cellular functions required during EMT or for the maintenance of mesenchymal states of cells, we employed a combination of quantitative proteomics and metabolomics. Of 5,574 proteins that were detected by mass spectrometry, 255 proteins were downregulated and 276 were upregulated in EGF-treated MDA-MB-468 cells (Fig. $3 a$ and Supplementary Table 1). Gene ontology revealed a bias towards proteins with oxidoreductase activity having an increased expression in the mesenchymal state (Extended Data Fig. 4a). The response to EGF was further characterized by changes in EMT markers, proteins linked to metastasis,

Fig. 1 | CD44 mediates Hyal-dependent iron endocytosis. a, Molecular structure (top) and ${ }^{1} \mathrm{H}$ NMR spectra (bottom) of LMM Hyal. Functional groups susceptible to reversibly interact with iron are highlighted in red. b. Western blots of CD44 and TfR1 in CD44 and TFRC ko clones generated using CRISPR-Cas9. c, Fluorescence microscopy of internalized Hyal-fluorescein isothiocyanate (FITC). $n=3$ biologically independent experiments. d, Fluorescence microscopy of RhoNox-M-positive vesicles colocalizing with internalized Hyal-FITC or TF-647. Dotted lines delineate cell contours. $n=3$ biologically independent experiments. e, Fluorescence microscopy of RhoNox-M-positive vesicles colocalizing with Hyal-FITC in a CD44 ko clone complemented with CD44. $n=3$ biologically independent experiments. f, SIMS imaging of cellular iron in a TFRC ko clone. Yellow lines delineate cell contours. $n=1$. At least 50 cells were quantified per condition. $\mathbf{g}$, ICP-MS of cellular iron for HMLER CD44high cells. $n=5$ biologically independent experiments. $\mathbf{h}$, Western blots of ferritin. Data representative of $n=8$ biologically independent experiments. $\mathbf{i}$, Schematic illustration (top) of CD44 high/low cell sorting from a PDX by flow cytometry. Western blots (bottom) of iron homeostasis proteins in CD44 ${ }^{\text {high/low }}$ cells sorted from breast cancer tumours. $n=1$. $\mathbf{b}-\mathbf{h}$, Parental HMLER CD44 ${ }^{\text {high }}$ and corresponding ko cell lines. Scale bars, $10 \mu \mathrm{m}$. f-h, HMM Hyal (0.6-1 MDa). Data representative of $n=3$ biologically independent experiments throughout the figure unless stated otherwise. Bars and error bars, mean values \pm s.d. c-e, One-way analysis of variance (ANOVA) with Bonferroni correction. g,h, Unpaired t-test. Statistical tests two-sided throughout the figure. See the Supplementary Information for full western blots. a.u., arbitrary units. DAPI, 4',6-diamidino-2-phenylindole; FAC, ferric ammonium citrate; PDX, patient-derived xenograft; TFA, trifluoroacetic acid. Unt, untreated. 
proteins involved in the regulation of endocytosis and iron homeostasis, as well as metabolic enzymes and an iron-dependent demethylase. For example, EMT was characterized by reduced levels of the epithelial proteins basal cell adhesion molecule (BCAM) and cell adhesion molecule 4 (CADM4), whereas vimentin was upregulated (Fig. 3a). The prometastatic cluster of differentiation 109 (CD109) was upregulated ${ }^{25}$, in agreement with the notion that mesenchymal cancer cells are capable of dissemination. Consistent with the a

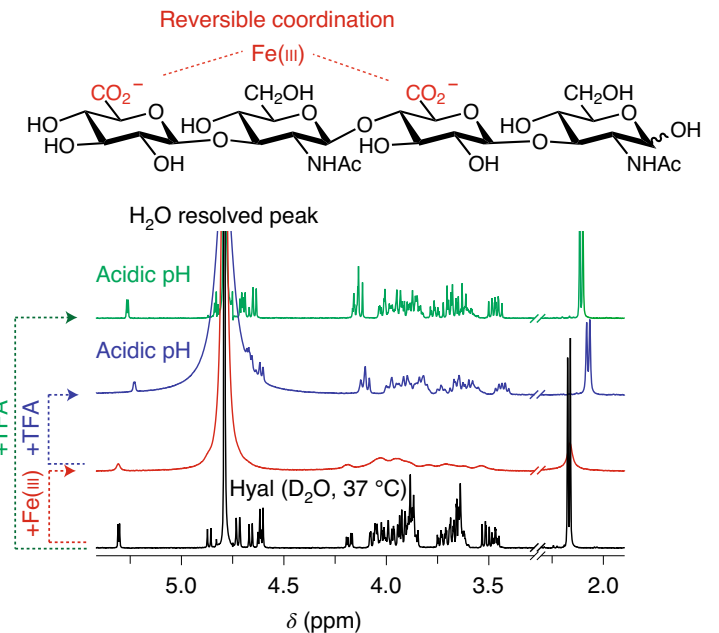

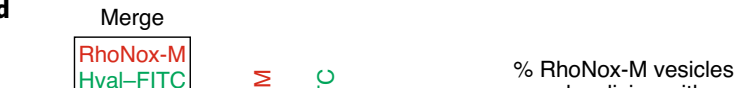
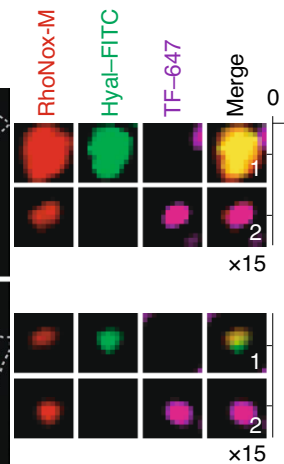

|ศึ่ำ colocalizing with

c
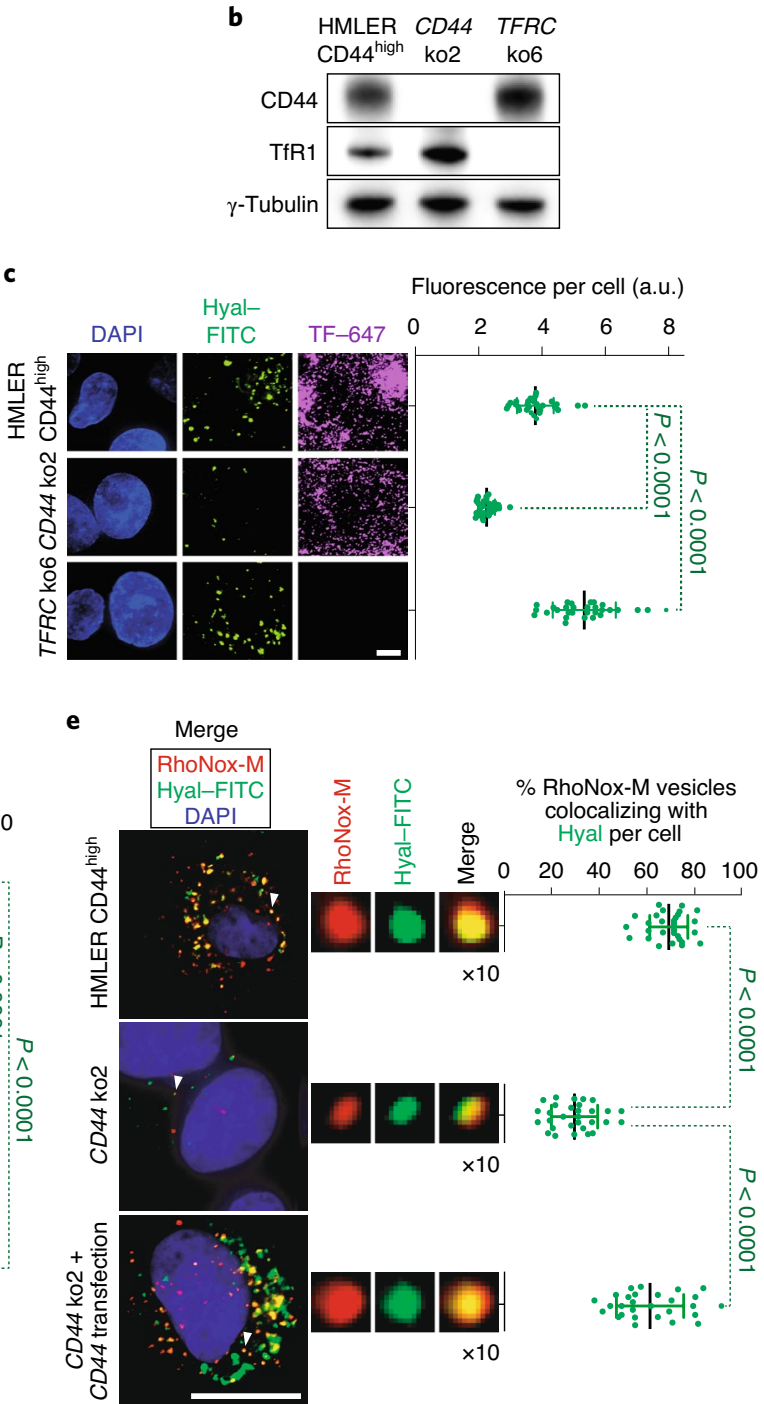

f

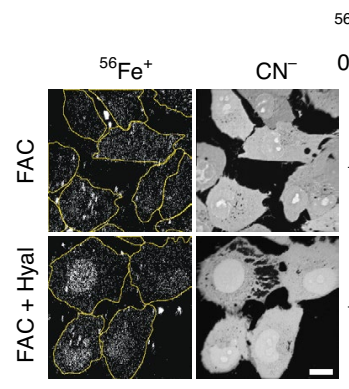

${ }^{56} \mathrm{Fe}$ content per cell (a.u.)

$\begin{array}{lllll}0.0 & 0.2 & 0.4 & 0.6 & 0.8\end{array}$

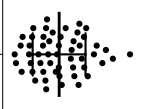

:

HMLER CD $44^{\text {high }}$

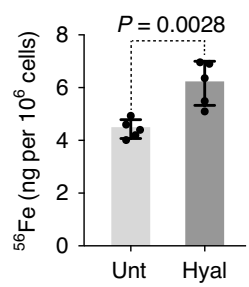

h HMLER

HMLER
CD44

Unt Hyal Unt Hyal Unt Hyal

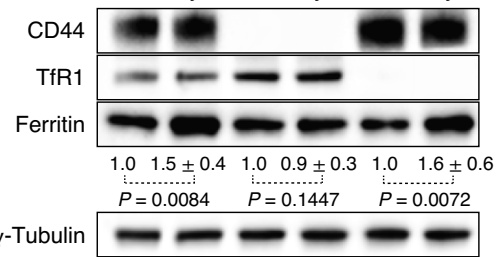

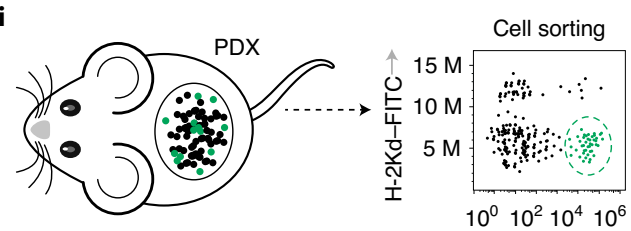

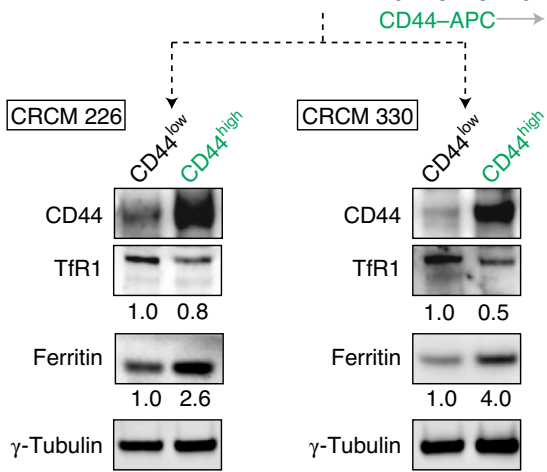


a
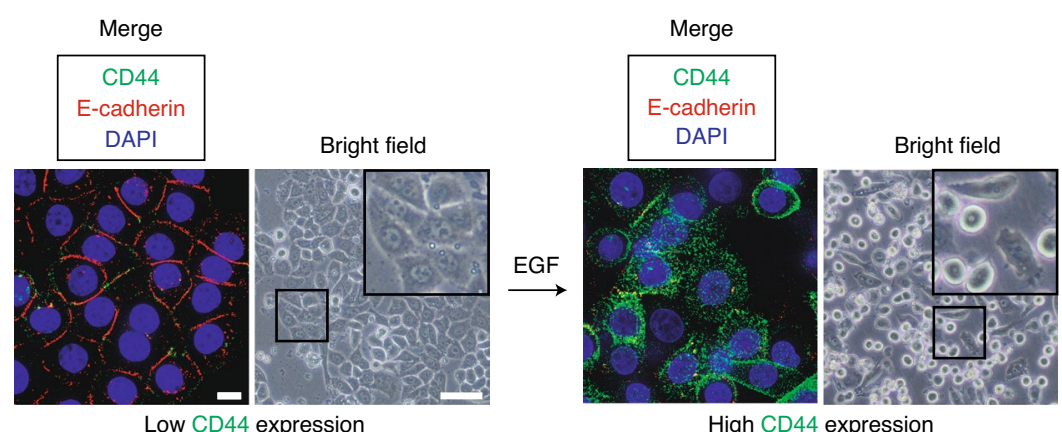

High CD44 expression
(1)

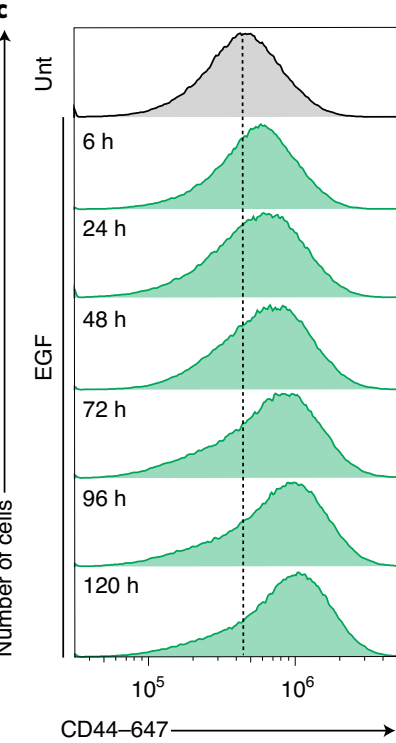

CD44-647

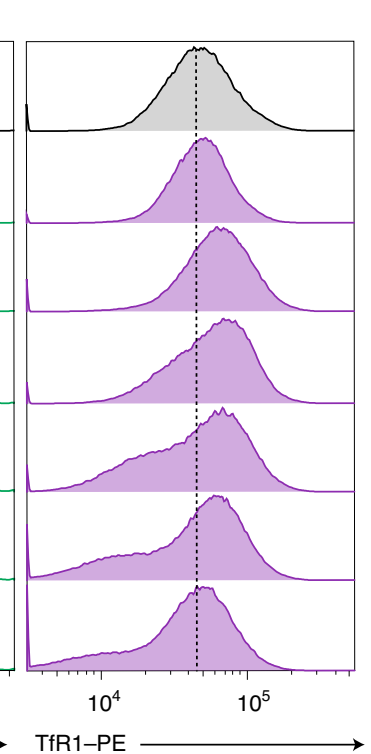

d

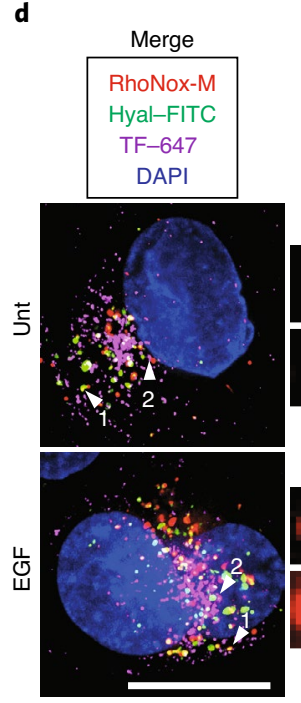

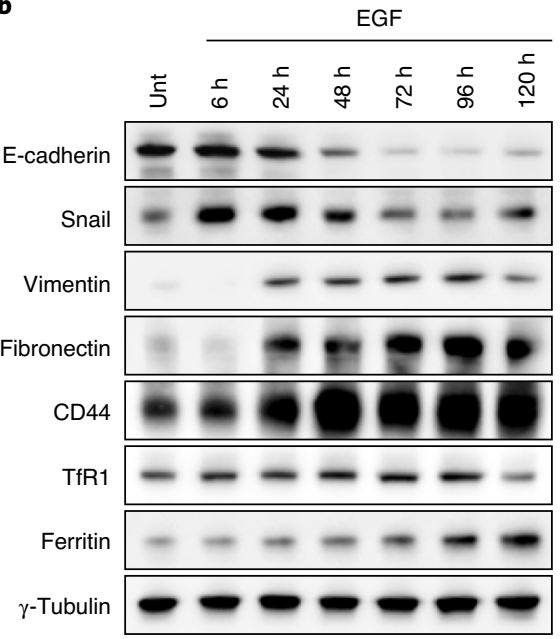

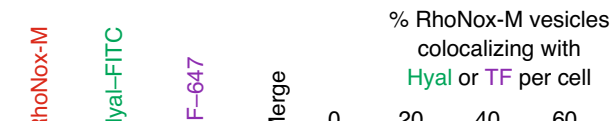

80

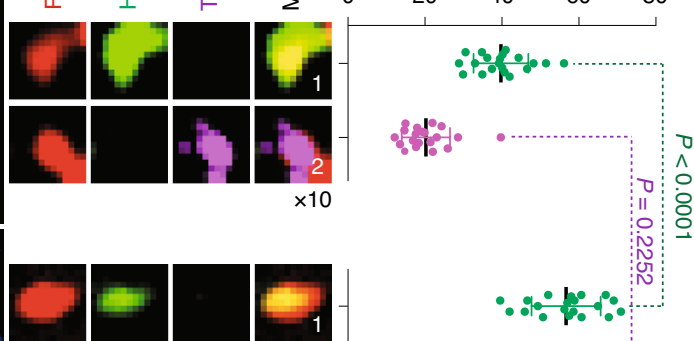

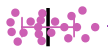
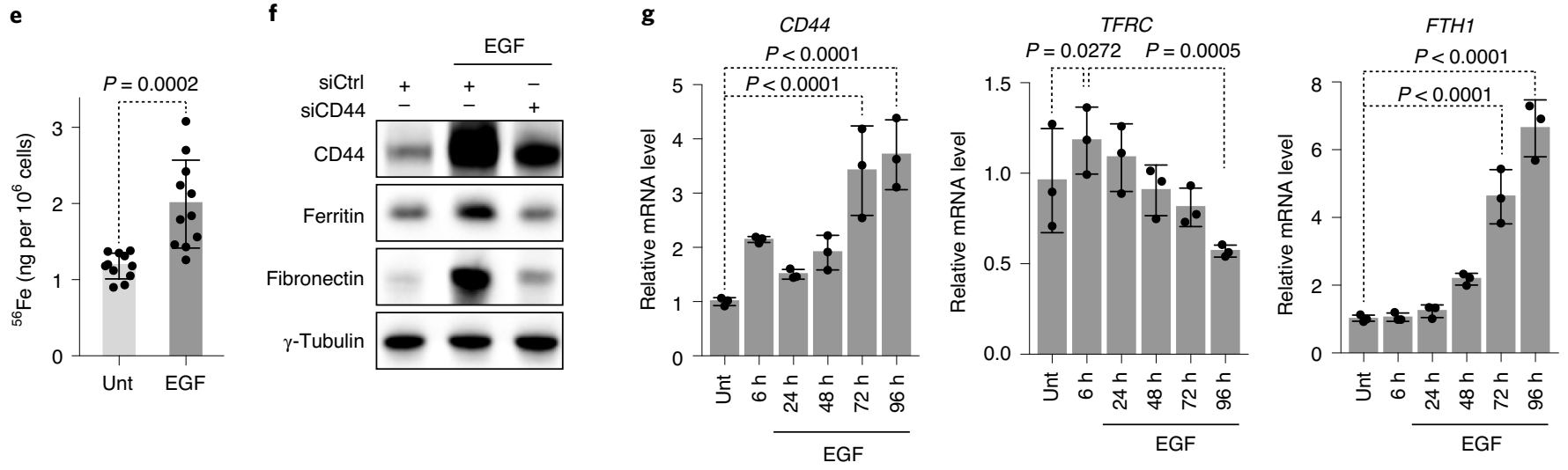

Fig. 2 | CD44-mediated iron endocytosis prevails in the mesenchymal state of cells. a, Fluorescence microscopy of EMT markers and bright-field microscopy of cell morphology. Scale bars, $10 \mu \mathrm{m}$ (left pair) and $100 \mu \mathrm{m}$ (right pair). b, Time-course western blots of iron homeostasis proteins and EMT markers. c, Time course flow cytometry of CD44 and TfR1 at the plasma membrane. d, Fluorescence microscopy of RhoNox-M-positive vesicles colocalizing with internalized Hyal-FITC or TF-647. Scale bar, $10 \mu \mathrm{m} . n=3$ biologically independent experiments. e, ICP-MS of cellular iron. $n=11$ biologically independent experiments. $\mathbf{f}$, Western blots of ferritin in CD44 knockdown conditions. $\mathbf{g}$, Time course RT-qPCR of CD44, TFRC and FTH1 messenger RNA (mRNA). $n=3$ biologically independent experiments. Results from MDA-MB- 468 cells are shown in the panels; the cells were treated with EGF for $72 \mathrm{~h}$ unless stated otherwise. Data representative of $n=3$ biologically independent experiments throughout the figure unless stated otherwise. Bars and error bars, mean values \pm s.d. One-way ANOVA with Bonferroni correction. d,e, Unpaired $t$-test. Statistical tests two-sided throughout the figure. See the Supplementary Information for full western blots. CD44-647, anti-CD44 antibody coupled to Alexa-647; siCD44, small interfering RNA against CD44; siCtrl, control small interfering RNA; TfR1-PE, anti-TfR1 antibody coupled to phycoerythrin. 


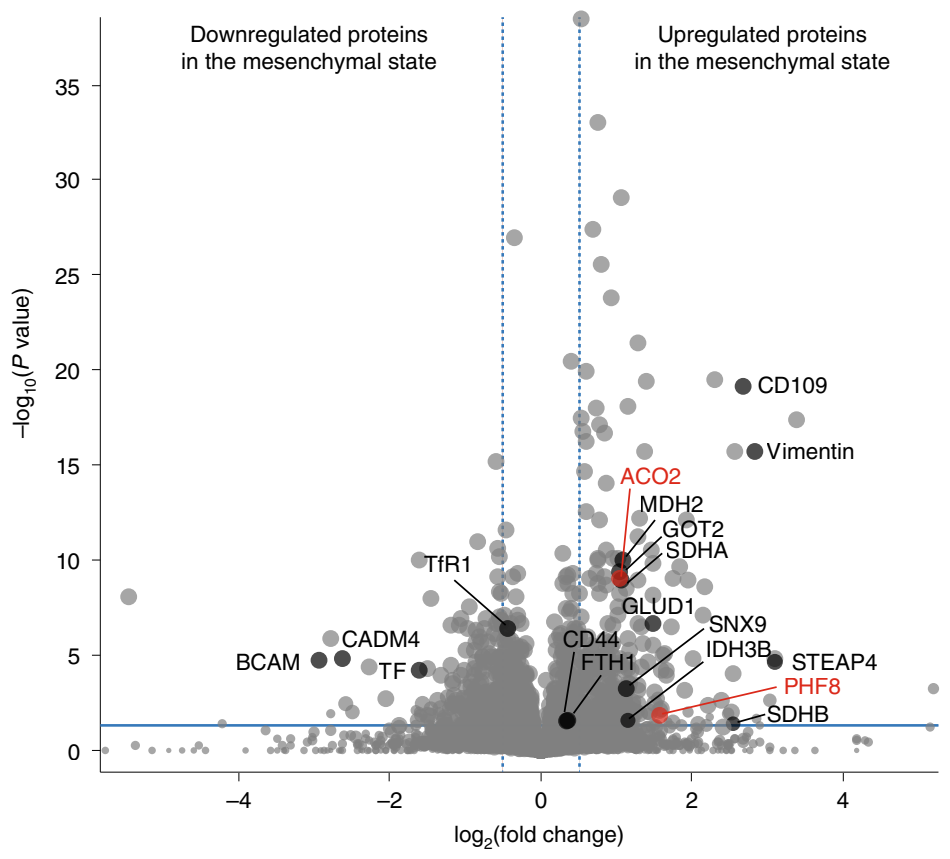

b

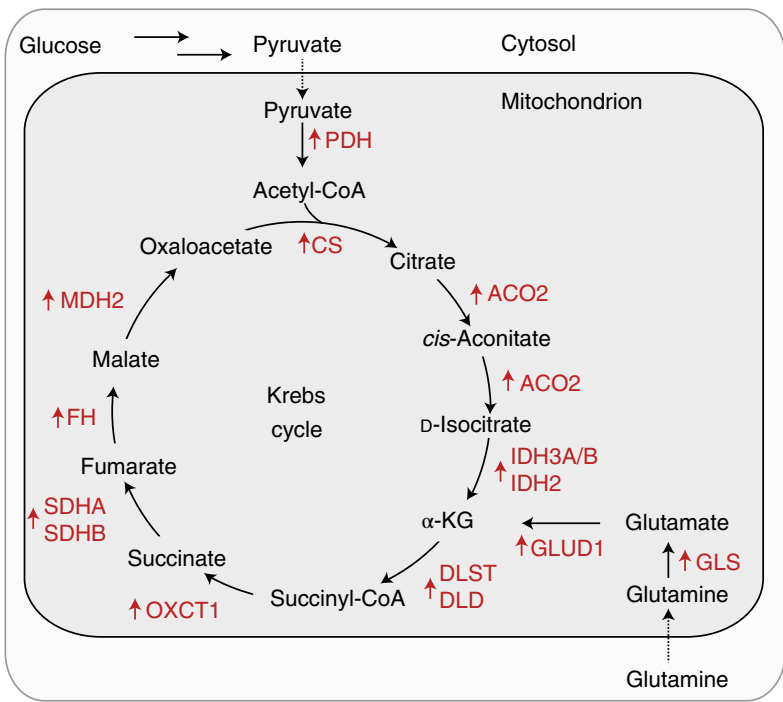

$\uparrow$ Upregulated enzyme in the mesenchymal state

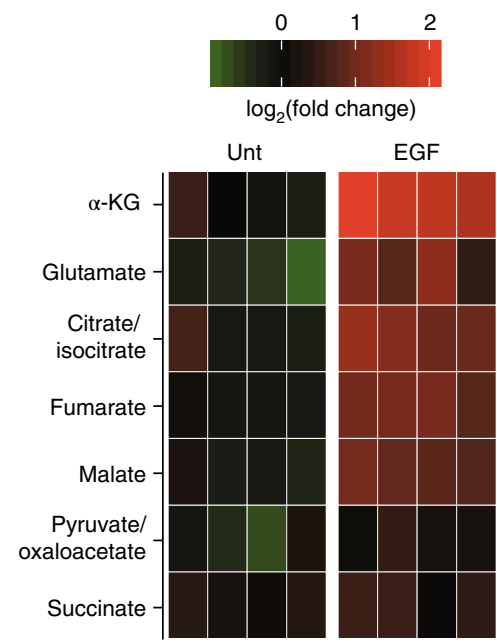

d

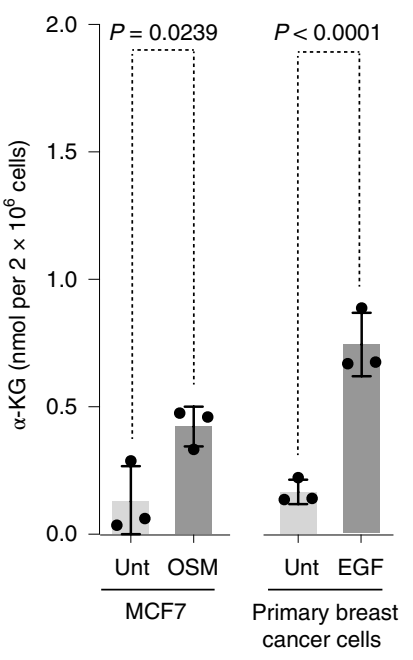

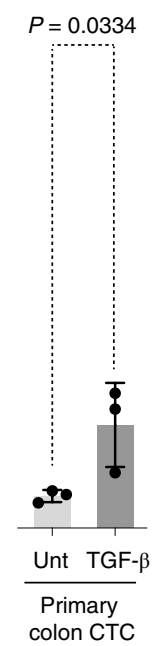

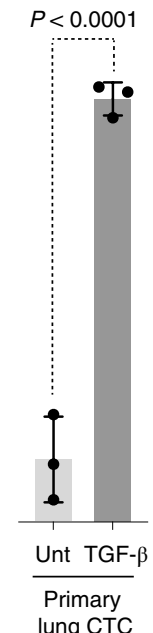

e

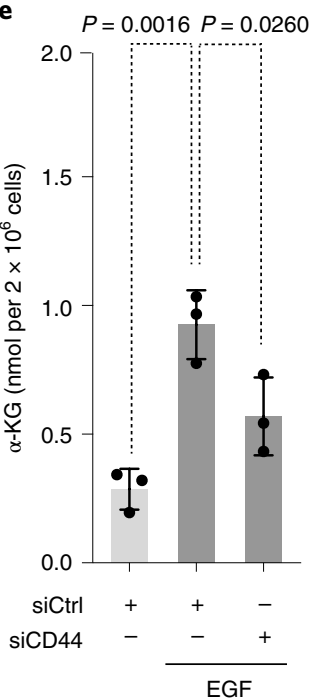

Fig. 3 | EMT is characterized by a redox signature that implicates iron. a, Quantitative label-free proteomics: a volcano plot of protein-expression fold changes (EGF versus Unt). Dashed blue line, fold change of 1.42; continuous blue line, $P$ value of 0.05 . The mitochondrial iron-sulfur cluster-containing protein ACO2 and the nuclear iron-dependent demethylase PHF8 are highlighted in red. $n=3$ biologically independent experiments. See the Supplementary Information for statistical analysis. b. Schematic illustration of metabolic pathways involved in the production of $\alpha$ KG. c, Quantitative metabolomics: heatmap of upregulated and downregulated metabolites in cells treated with EGF for $60 \mathrm{~h} . n=4$ technical replicates. $\mathbf{d}$, $\alpha$ KG quantification assay. Cells treated as indicated for 72 h. $n=3$ technical replicates. e, $\alpha K G$ quantification assay in CD44 knockdown conditions. $n=3$ biologically independent experiments. Unpaired t-test. a-c,e, MDA-MB-468 cells were used and treated with EGF for $72 \mathrm{~h}$ unless stated otherwise. Bars and error bars, mean values \pm s.d. e, One-way ANOVA with Bonferroni correction. Statistical tests two-sided throughout the figure. CoA, co-enzyme A; CS, citrate synthase; DLD, dihydrolipoamide dehydrogenase; DSLT, dihydrolipoamide S-succinyltransferase; FH, fumarate hydratase; FTH1, ferritin heavy chain 1; GLS, glutaminase; GOT2, glutamic oxaloacetic transaminase 2; IDH3B, isocitrate dehydrogenase (NAD(+)) 3 beta; MDH2, malate dehydrogenase 2; OXCT1, 3-oxoacid CoA-transferase 1; PDH, pyruvate dehydrogenase; SDHA/SDHB, succinate dehydrogenase subunit A/B; STEAP4, six-transmembrane epithelial antigen of the prostate 4.

upregulation of iron uptake during EMT, levels of ferritin increased together with those of sorting nexin 9 (SNX9), a protein that regulates endocytosis of CD44, which has also been linked to metastasis $^{26}$. Conversely, levels of TF and TfR1 were reduced, which further supports the prevalent functional role of CD44 in mediating iron endocytosis during EMT. Importantly, we detected an increase of the iron-dependent histone demethylase plant homeodomain finger protein 8 (PHF8), which is involved in active transcription, brain development and cell cycle progression, as well as the regulation of EMT in vivo and in breast tumour growth ${ }^{27-31}$. In contrast, levels of other iron-dependent demethylases that could be detected by mass spectrometry did not change substantially. Western blotting further supported protein changes identified by mass spectrometry (Extended Data Fig. 4b). 
Additionally, metabolic enzymes involved in the conversion of glutamate and pyruvate into $\alpha$-ketoglutarate $(\alpha \mathrm{KG})$, which include glutamate dehydrogenase 1 (GLUD1), isocitrate dehydrogenase 2 (IDH2) and the iron-sulfur cluster-containing aconitase 2 (ACO2), were upregulated along with other enzymes of the Krebs cycle (Fig. 3a,b). $\alpha \mathrm{KG}$ is a cosubstrate required for the iron-catalysed oxidative demethylation of histone and nucleic acid methyl marks, and is implicated in the maintenance of pluripotency ${ }^{32}$. Consistent with the upregulation of these mitochondrial enzymes, quantitative metabolomics indicated a marked increase of $\alpha \mathrm{KG}$ in various cell lines and primary cells that undergo EMT (Fig. 3c,d, Extended Data Fig. 4c and Supplementary Table 2). We then investigated whether reducing iron uptake could potentially alter the mitochondrial metabolism in cells treated with EGF. Knocking down CD44 antagonized the upregulation of $\alpha \mathrm{KG}$ in cells treated with EGF (Fig. 3e), which was consistent with previous findings that showed iron restriction inhibits the activity of $\mathrm{ACO} 2^{33}$, which supports a role for iron in mitochondria during EMT.

These biochemical changes, which include an enhanced cellular uptake of iron together with the upregulation of PHF8 and its cosubstrate $\alpha \mathrm{KG}$, further hint at a specific regulation of EMT that occurs at the chromatin level ${ }^{34}$.

Nuclear iron is a rate-limiting regulator of epigenetic plasticity. Metals are ubiquitous in cell biology, acting as protein cofactors. Owing to its unique electron configuration, iron distinguishes itself by its capacity to catalyse oxidative demethylation of protein residues, which include those of histones, and methylated nucleobases. Thus, iron is a rate-limiting factor of these processes (Fig. 4a). In line with the role of iron in the nucleus of cells undergoing EMT, subcellular fractionation indicated higher levels of ferritin in the nucleus of MDA-MB-468 cells in the mesenchymal state (Fig. 4b) ${ }^{35}$.

The iron- and $\alpha \mathrm{KG}$-dependent demethylase PHF8 has been shown to promote demethylation of histone $\mathrm{H} 3$ lysine 9 dimethyl $(\mathrm{H} 3 \mathrm{~K} 9 \mathrm{me} 2)^{36}$, a repressive histone mark whose reduction has been observed in solid tumours ${ }^{37-39}$. The increase of PHF8 in MDA-MB-468 cells undergoing EMT correlated with the preferential reduction of $\mathrm{H} 3 \mathrm{~K} 9 \mathrm{me} 2$ compared with the other repressive histone marks histone $\mathrm{H} 3$ lysine trimethyl (H3K9me3) and histone $\mathrm{H} 3$ lysine 27 trimethyl (H3K27me3), as defined by quantitative mass spectrometry (Fig. 4c and Supplementary Table 3). Additionally, knocking down PHF8 prevented demethylation of H3K9me2 in MDA-MB-468 cells treated with EGF (Fig. 4 d), which validates a functional role for this demethylase during EMT.

Next, we examined the genome-wide distribution of H3K9me2 by chromatin immunoprecipitation sequencing (ChIP-seq) to identify PHF8-regulated genes whose expressions are iron-dependent. ChIP-seq revealed large organized heterochromatin K9 modifications, previously termed LOCKs ${ }^{37,39}$. These data showed a reduction of H3K9me2 ChIP-seq read counts in the body of specific genes of cells treated with EGF, which include CD44, CD109, VIM (coding for vimentin) and FN1 (coding for fibronectin) (Fig. 4e, Extended Data Fig. 5a and Supplementary Table 4), which was further validated by
ChIP-qPCR (Extended Data Fig. 5b). These data indicate that CD44 positively regulates its own expression at the transcriptional level by mediating iron endocytosis, unlike TfR1, which is negatively regulated by excess iron at the translational level via iron responsive elements ${ }^{23,24}$. Furthermore, we analysed the transcriptome by RNA-seq and found that the reduction of $\mathrm{H} 3 \mathrm{~K} 9 \mathrm{me} 2$ read counts in gene bodies correlated with increased gene expression, in agreement with the repressive nature of $\mathrm{H} 3 \mathrm{~K} 9 \mathrm{me} 2$ (Fig. $4 \mathrm{f}$ and Supplementary Table 4). For instance, of 284 genes for which the reduction of H3K9me2 was observed, 258 genes were transcriptionally upregulated. In addition to $C D 44$, we identified genes involved in cancer, regulation of EMT, development, immune responses, inflammation and wound healing, which are biological processes CD44 is linked to (Supplementary Table 5). In comparison, we did not observe a substantial reduction of $\mathrm{H} 3 \mathrm{~K} 9 \mathrm{me} 2$ in genes that code for EMT transcription factors. However, we identified long non-coding (lnc) RNA genes previously reported to be involved in cancer (Supplementary Table 5). In agreement with PHF8 playing a crucial role in regulating these genes through the oxidative demethylation of $\mathrm{H} 3 \mathrm{~K} 9 \mathrm{me} 2$, knocking down this demethylase partly blocked the EGF-induced expression of iron-regulated genes, which included CD44 (Fig. 4g). Consistently, ChIP-seq indicated that $\mathrm{H} 3 \mathrm{~K} 4 \mathrm{me} 3$ was enriched at active promoters, which is required for the recruitment and selective activity of PHF8 at these genomic loci (Extended Data Fig. 5c). Furthermore, ChIP-seq also revealed a reduction of the repressive histone mark H3K27me3 in a more restricted set of genes (Extended Data Fig. 5d), which was consistent with the increased mRNA levels of the H3K27-specific iron-dependent lysine demethylases 6A and $6 \mathrm{~B}$ (KDM6A and KDM6B) in cells undergoing EMT, as monitored by RNA-seq (Supplementary Table 4). Conversely, we did not observe changes in the heterochromatin mark H3K9me3 (Fig. 4c and Extended Data Fig. 5e). Although the reduction of H3K27me3 was less pronounced compared with that of $\mathrm{H} 3 \mathrm{~K} 9 \mathrm{me} 2$, according to mass spectrometry and ChIP-seq read counts (Fig. 4c and Extended Data Fig. 5d), these data support the central role of iron in the regulation of gene expression and reflect a complex scenario that occurs at the chromatin level during EMT, which potentially involves other iron-dependent demethylases and histone marks. In line with this, knocking down CD44 partly blocked H3K9me2 demethylation and the expression of genes regulated by PHF8 in MDA-MB-468 cells (Fig. 4h,i). Consistently, CD44 ${ }^{\text {high }}$ cells from tumours exhibited higher levels of PHF8 and the iron-regulated gene fibronectin with reduced $\mathrm{H} 3 \mathrm{~K} 9 \mathrm{me} 2$ compared with those of the $\mathrm{CD} 44^{\text {low }}$ population, which supports the physiological relevance of this mechanism (Fig. 4j). Taken together, these data establish a direct functional link between CD44-mediated iron endocytosis and the regulation of gene expression that involves iron-catalysed histone demethylation.

Targeting iron homeostasis interferes with the maintenance of a mesenchymal state of cells. Small molecules provide a means to manipulate cellular processes with valuable spatial and temporal resolution ${ }^{40}$. To directly address the implication of the nuclear iron pool, we set out to identify small-molecule chelators of iron that

Fig. 4 | Nuclear iron is a rate-limiting regulator of epigenetic plasticity. a, Catalytic cycle of iron-mediated oxidative demethylation of a methylated lysine residue. b, Subcellular fractionation and western blots of nuclear ferritin. c, Quantitative mass spectrometry: heatmap of H3K9me2, H3K9me3 and H3K27me3. $n=5$ biologically independent experiments. Two-sided associated $t$-tests. d, Western blots of H3K9me2 in PHF8 knockdown conditions. e, H3K9me2 ChIP-seq profiles for selected genes. $\mathbf{f}$, Scatter plot correlation of H3K9me2 ChIP-seq read counts in gene bodies ( $n=3$ biologically independent experiments) and RNA-seq ( $n=3$ biologically independent experiments). $\mathbf{g}$, Western blots of proteins whose genes are regulated by H3K9me2 in PHF8 knockdown conditions. h, Western blots of H3K9me2 in CD44 knockdown conditions. $\mathbf{i}$, Western blots of proteins whose genes are regulated by H3K9me2 in CD44 knockdown conditions. j, Western blots of fibronectin, PHF8 and H3K9me2 in CD44high/low cells sorted from breast cancer tumours. $n=1$. b-i, MDA-MB-468 cells used for and treated with EGF for $72 \mathrm{~h}$. Data representative of $n=3$ biologically independent experiments unless stated otherwise. See the Supplementary Information for statistical analyses and full western blots. CDCP1, CUB domain-containing protein 1; $\mathrm{CE}$, cytoplasmic extract; FSCN1, fascin actin-bundling protein; Kme2, dimethyllysine; MGLL, monoglyceride lipase; NE, nuclear extract; siPHF8, small interfering RNA against PHF8; WCE, whole-cell extract. 
a

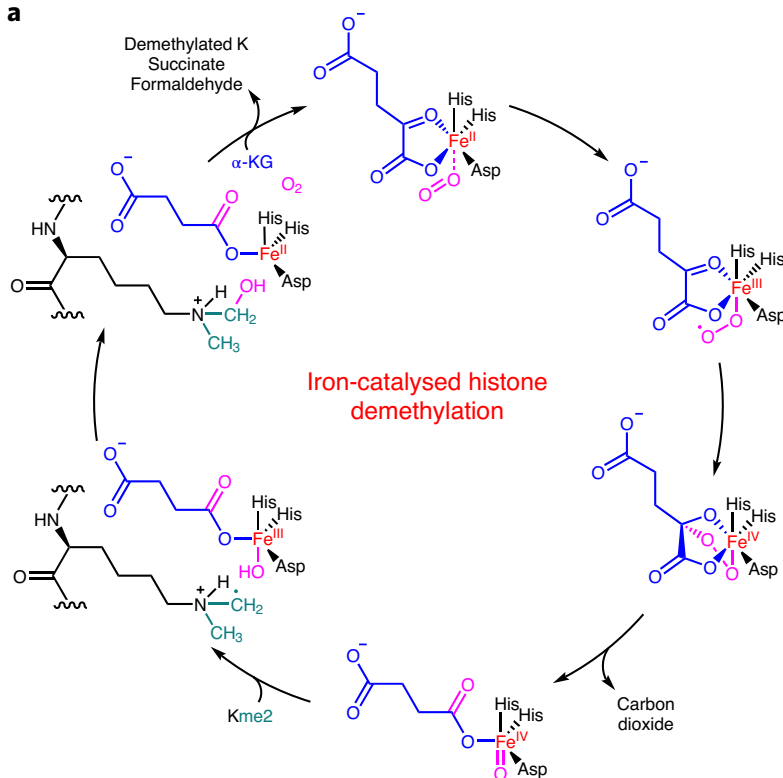

b

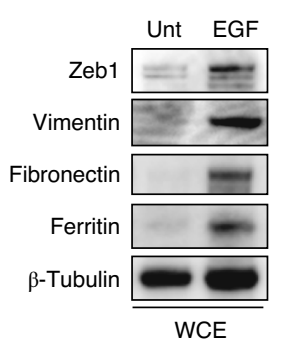

c

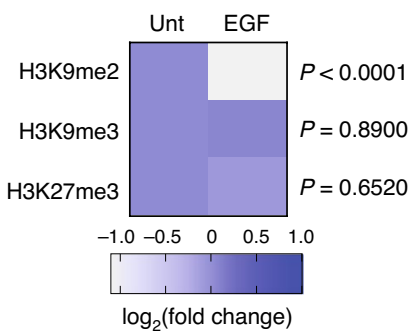

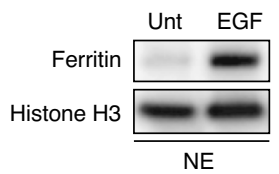

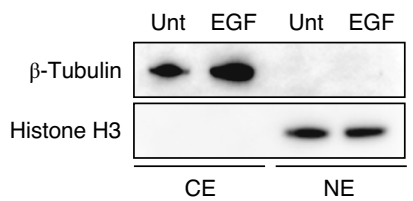

d

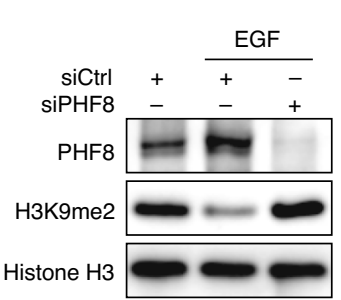

e

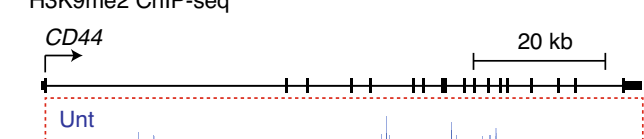

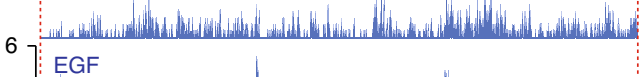

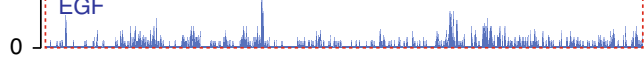
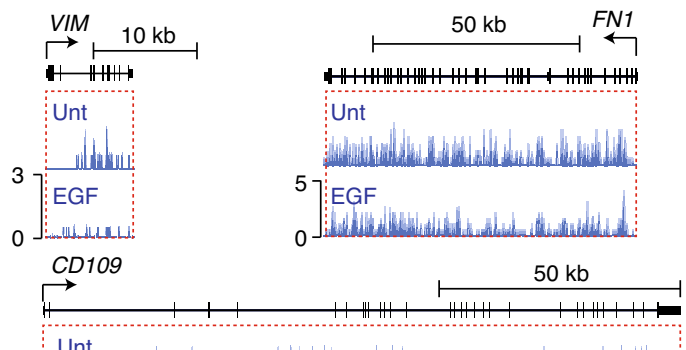

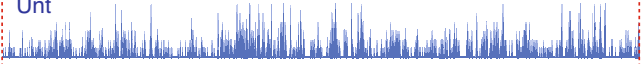

3 EGF

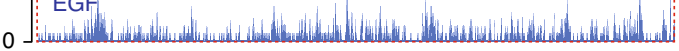

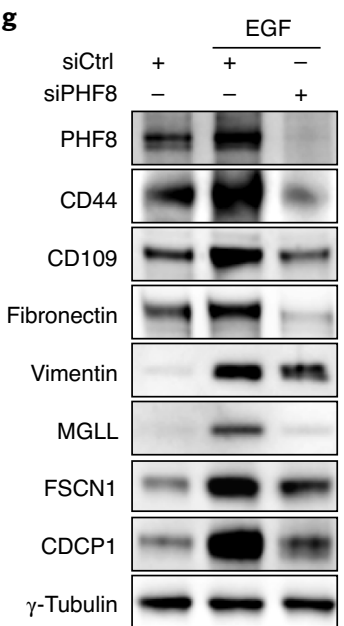

h

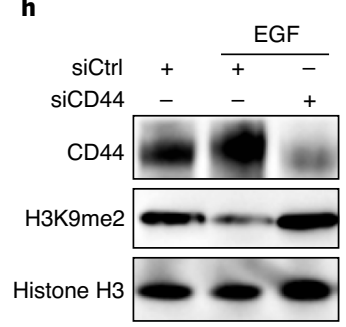

f
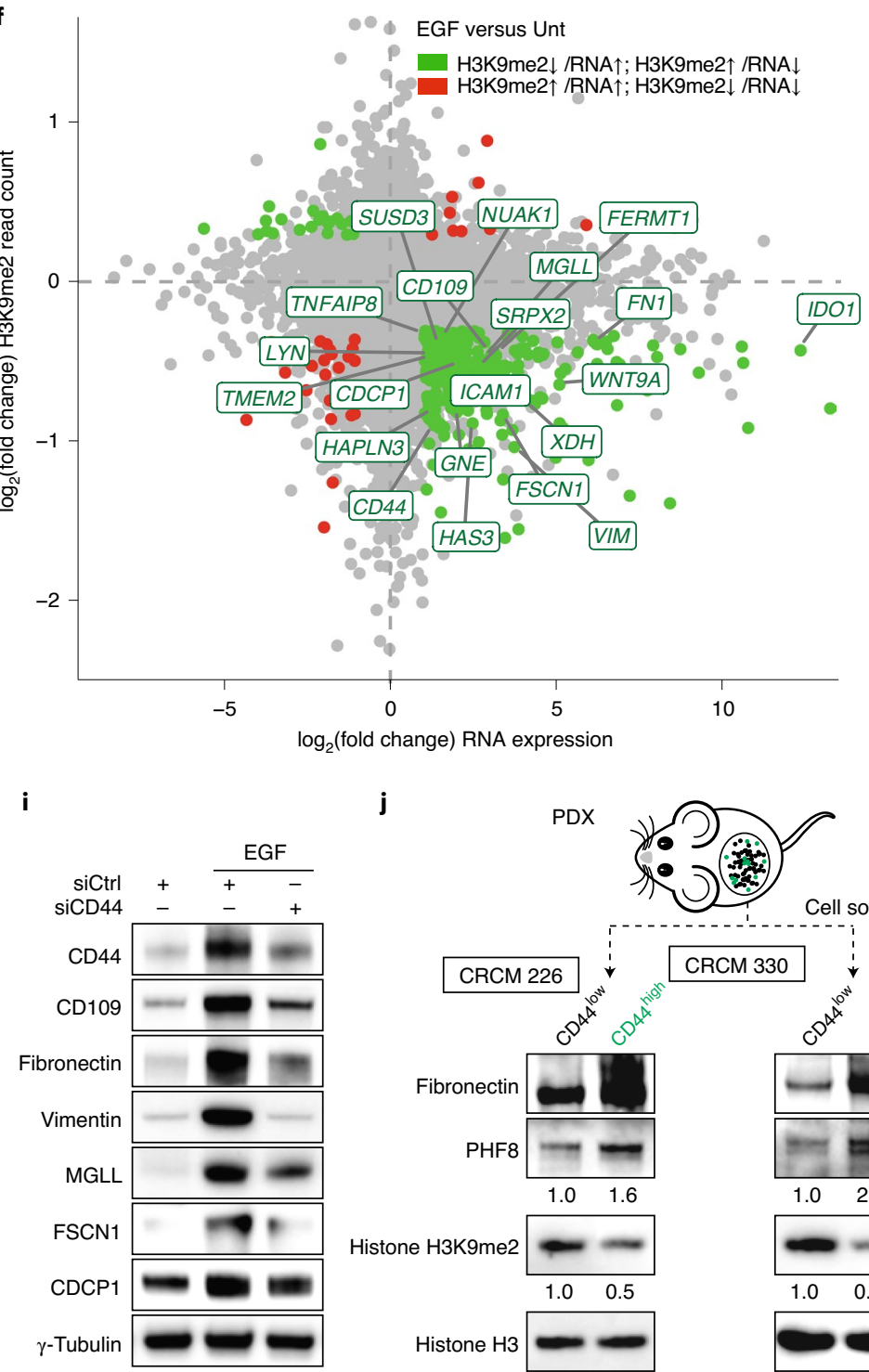

j

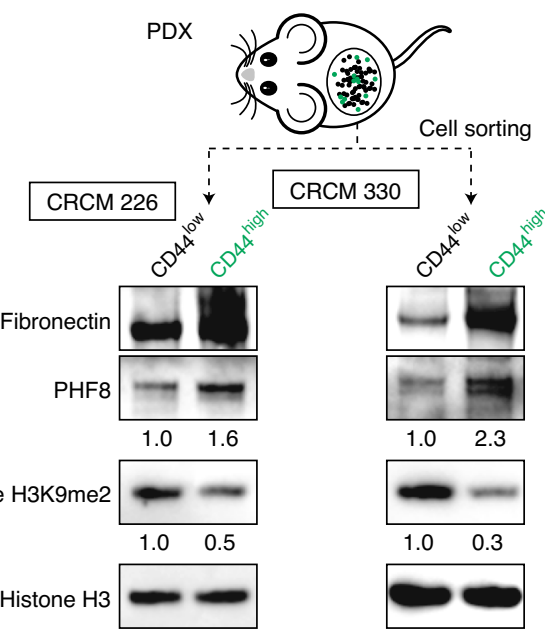


a

Click

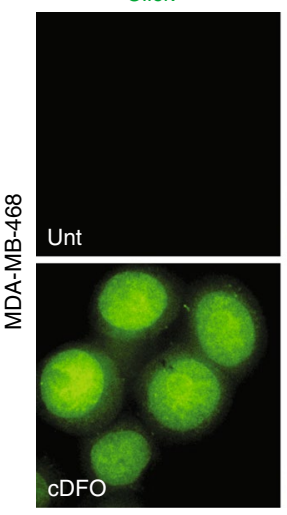

DAPI

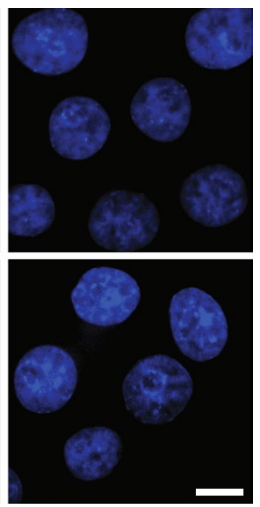

b

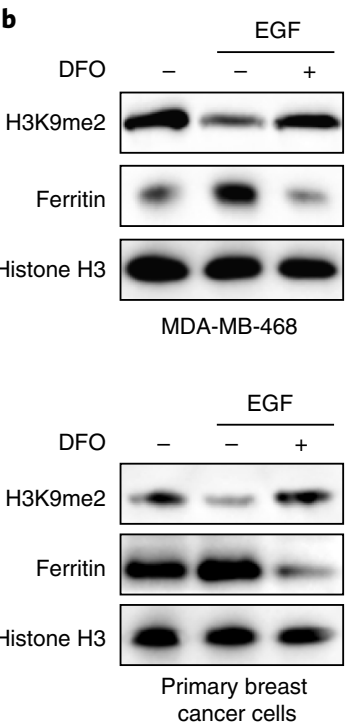

e

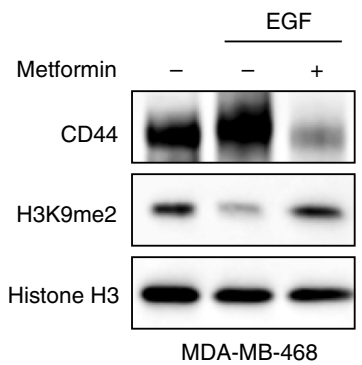

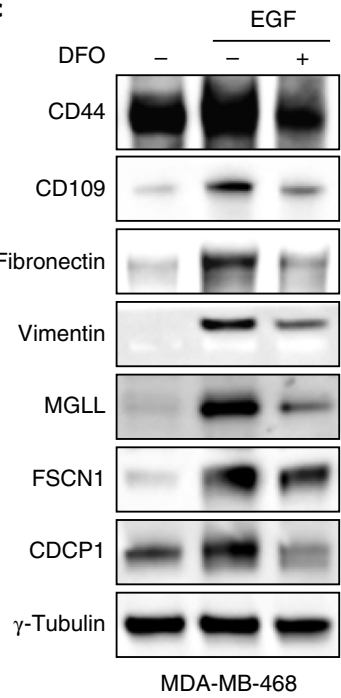

f

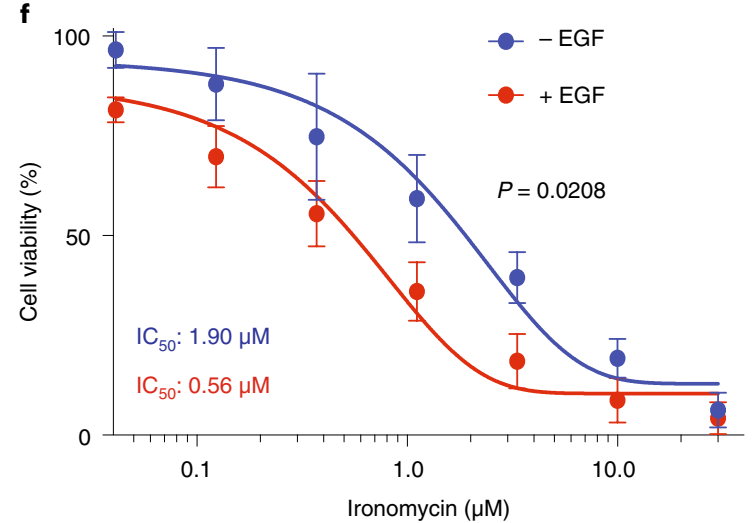

d
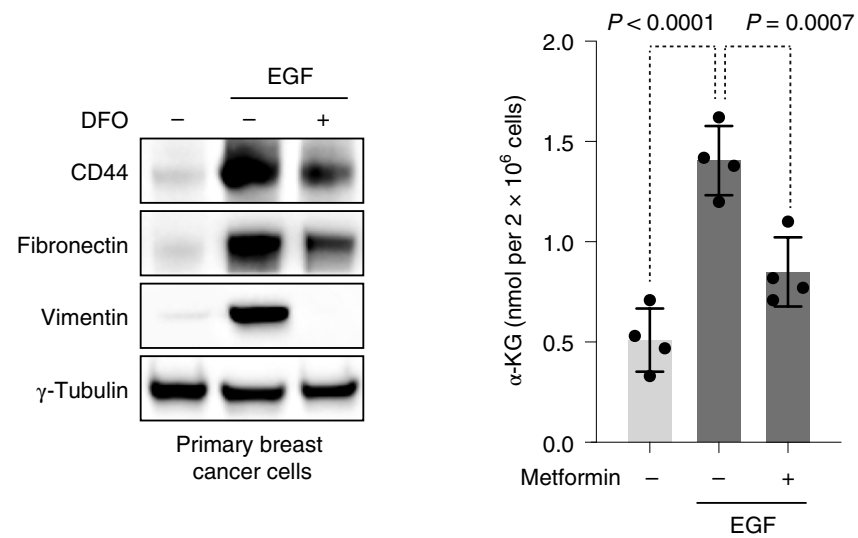

g

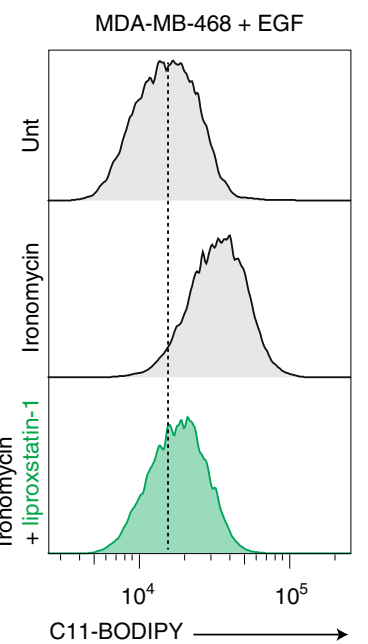

Fig. 5 | Targeting iron homeostasis interferes with the maintenance of mesenchymal cells. a, Molecular structure of cDFO (top), schematic illustration of chemical labelling of cDFO in cells (bottom) and fluorescence microscopy images of labelled cDFO and DAPI (right). 488 represents Alexa-488.

Scale bar, $10 \mu \mathrm{m}$. b. Western blots of H3K9me2 in cells co-treated with EGF and DFO. $n=1$ for primary cells. c, Western blots of proteins whose genes are regulated by H3K9me2 in cells co-treated with EGF and DFO. $n=1$ for primary cells. $\mathbf{d}$, $\alpha$ KG quantification assay for MDA-MB- 468 cells. $n=4$ biologically independent experiments. One-way ANOVA with Bonferroni correction. e, Western blots of H3K9me2 in cells co-treated with EGF and metformin. f, Viability curves of MDA-MB-468 cells co-treated with EGF and ironomycin. $n=4$ biologically independent experiments. Two-way ANOVA with Bonferroni correction. $\mathbf{g}$, Flow cytometry analysis of C11-BODIPY in cells treated as indicated. $n=1$. Data representative of $n=3$ biologically independent experiments throughout the figure unless stated otherwise. Bars and error bars, mean values \pm s.d. Statistical tests two-sided throughout the figure. See the Supplementary Information for full western blots. $I_{50}$, half-maximum inhibitory concentration.

could specifically target the nucleus. To this end, we functionalized known iron chelators with alkyne functional groups that can be chemically labelled in cells by means of click chemistry (Fig. $5 \mathrm{a})^{41}$.
Fluorescent labelling of a clickable surrogate of deferoxamine (cDFO) in cells revealed a predominant nuclear localization of this small molecule (Fig. 5a). Thus, deferoxamine (DFO) represents 


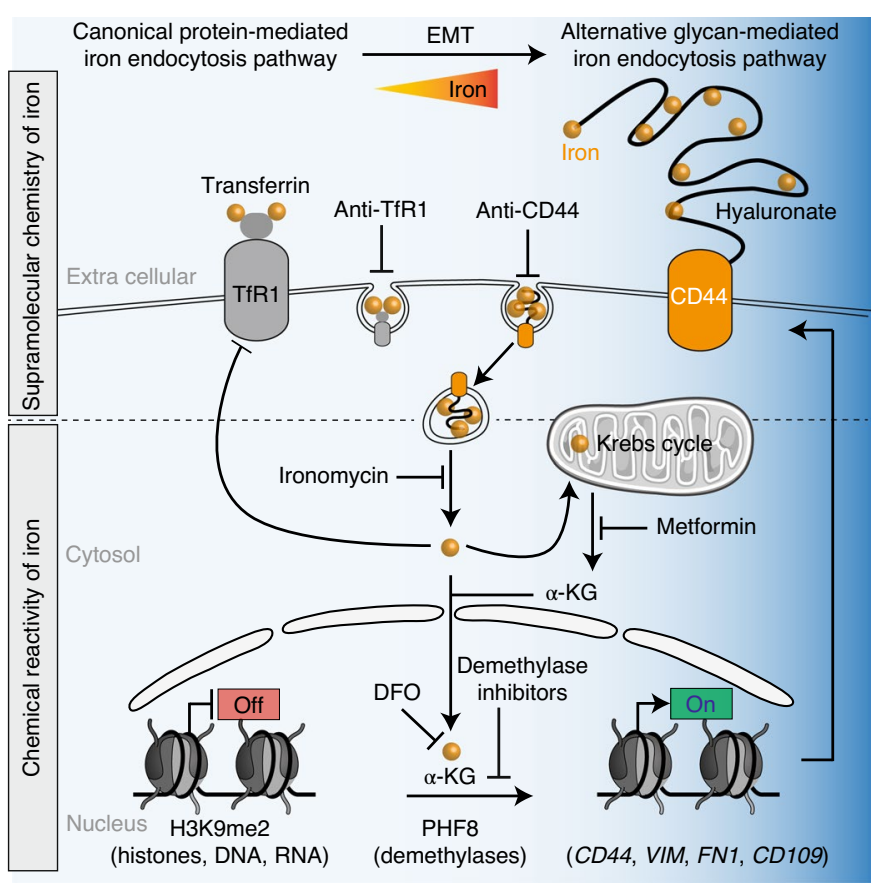

Fig. 6 | Reciprocal endocytic-epigenetic regulation that involves iron. TF or iron-bound Hyal enters cells by means of TfR1- or CD44-mediated endocytosis. Excess cellular iron inhibits the canonical TfR1 pathway. Nuclear iron, $\alpha K G$ produced in mitochondria and iron-dependent enzymes mediate the oxidative demethylation of chromatin marks to unlock the expression of specific genes including CD44. CD44 positively regulates its own expression at the transcriptional level by mediating iron endocytosis and this pathway prevails in the mesenchymal state of cells. Iron homeostasis can be targeted at the plasma membrane, the lysosomal compartment, the mitochondria and in the nucleus using specific antibodies or small molecules.

a suitable tool to directly interrogate the functional roles of nuclear iron. For instance, DFO prevented the demethylation of $\mathrm{H} 3 \mathrm{~K} 9 \mathrm{me} 2$ in cancer cell lines and primary cancer cells that were treated with EGF, OSM or TGF- $\beta$ (Fig. 5b and Extended Data Fig. 6a). Consistently, DFO antagonized the upregulation of proteins whose genes were found to be regulated by PHF8 in these conditions (Fig. $5 \mathrm{c}$ and Extended Data Fig. 6b). These data provide direct evidence of the functional role of nuclear iron in the regulation of the expression of these genes. Moreover, the iron chelator deferasirox, whose scaffold exhibits a preference for the mitochondrial compartment, as defined by the staining of the clickable derivative (Extended Data Fig. $6 c$ ), blocked $\alpha$ KG production in MDA-MB-468 cells treated with EGF (Extended Data Fig. 6d) and interfered with H3K9me2 demethylation and CD44 expression (Extended Data Fig. 6e). Similarly, metformin, which has been shown to target metals in the mitochondria ${ }^{42}$ and to inhibit the Krebs cycle ${ }^{43}$, antagonized the effect of EGF (Fig. $5 \mathrm{~d}, \mathrm{e}$ ). These data, consistent with $\alpha \mathrm{KG}$ being required for the iron-dependent demethylation of $\mathrm{H} 3 \mathrm{~K} 9 \mathrm{me} 2$, validate a functional role for mitochondrial iron during EMT and show that targeting the mitochondrial metabolism provides another means to control epigenetic plasticity.

Finally, the lysosomal iron-sequestering small-molecule ironomycin has previously been shown to effectively kill mesenchymal and tumour-initiating cells in vitro and in vivo due to a higher iron content in these cells ${ }^{15}$. Consistently, EGF potentiated the cytotoxic effect of ironomycin against MDA-MB-468 cells (Fig. 5f) with increased lipid peroxidation, as defined by C11-BODIPY fluorescence that was prevented by co-treatment with liproxtatin-1 (Fig. $5 g)^{44}$.
These data are in line with the observed increase of iron endocytosis in these conditions and provide a powerful strategy to eradicate tumorigenic cells undergoing EMT through the induction of ferroptosis ${ }^{45}$. Together, these data support a mechanism whereby iron is a rate-limiting regulator of epigenetic plasticity ${ }^{46}$, a general pathway prone to small-molecule intervention with topological resolution.

\section{Discussion}

This investigation illuminates a unifying mechanism that involves CD44, Hyal and iron in the regulation of epigenetic plasticity (Fig. 6). Our findings link iron endocytosis to an enhanced mitochondrial metabolism and molecular changes that occur at the chromatin level. The free carboxylates of Hyal, which is a principal ligand of CD44, are prone to interact with iron outside cells at physiological pH, and thereby enable CD44 to mediate endocytosis of these organometallic complexes. On acidification of endocytic vesicles and the ensuing protonation of Hyal, iron is released and translocates into the cytosol to traffic towards specific cellular compartments. In particular, increased levels of nuclear ferritin and upregulation of the iron-dependent demethylase PHF8 together with the reduction of the repressive histone mark $\mathrm{H} 3 \mathrm{~K} 9 \mathrm{me} 2$ in an iron-dependent manner provide solid evidence for a functional role of nuclear iron during EMT. Upregulation of other demethylases and detectable alterations of other histone marks reflect a complex regulation at the chromatin level that is iron-dependent.

In this context, two independent iron endocytosis pathways co-exist, one reliant on the protein TF and the other on the glycan Hyal (Fig. 6). Interestingly, these biomolecules represent distinct classes of organic metal carriers of different biosynthetic origins. The CD44-dependent pathway that involves Hyal is switched on at the onset of EMT, and progressively takes over as the prevalent mechanism of iron endocytosis compared with the TfR1-dependent pathway. Importantly, CD44 is an iron-regulated gene, which indicates a positive feedback loop whereby CD44 regulates its own expression. In contrast, TfR 1 is negatively regulated at the translational level by excess iron. This provides a rationale that underlies the global increase of CD44 and the reduction of TfR1 during the course of EMT. In epithelial cells, the iron demand is moderate, which makes the self-regulated TfR1-dependent pathway sufficient to maintain a basal level of iron. However, the higher needs of iron in the mesenchymal state of cells to unlock the expression of mesenchymal genes cannot be solely fulfilled by this pathway. Thus, upregulation of CD44 triggered by growth factors, cytokines and other signalling molecules represents a powerful alternative to increase the cellular iron load.

In the nucleus, using $\alpha \mathrm{KG}$ and taking advantage of demethylases, which include PHF8, iron operates as a metal catalyst that mediates oxidative demethylation of histone residues. Iron is therefore a rate-limiting regulator of the expression of specific genes (Fig. 6). Hence, to unlock these genes, cells concomitantly upregulate the production of demethylases and increase iron uptake. The repressive histone mark $\mathrm{H} 3 \mathrm{~K} 9 \mathrm{me} 2$, which is a direct substrate of iron and PHF8, was identified as a key post-translational modification that regulates the expression of mesenchymal genes. However, other enzymes involved in methylation, acetylation and deacetylation are expected to work in concert with this iron-dependent demethylation of $\mathrm{H} 3 \mathrm{~K} 9 \mathrm{me} 2$. Our analysis revealed that $\mathrm{H} 3 \mathrm{~K} 9 \mathrm{me} 2$ governs the expression of genes involved in cancer, development, immune responses, inflammation and wound healing. Interestingly, CD44 has previously been linked to these processes, consistent with the regulatory role of iron.

Cellular iron homeostasis can be altered at the plasma membrane by interfering with iron endocytosis using specific antibodies, or alternatively by controlling the chemical reactivity of this metal in selected cellular compartments using the appropriate small molecules (Fig. 6). These pharmacological tools provide a means to dissect the processes reliant on iron in various settings and can be further developed for therapeutic intervention. 
Intriguingly, Hyal with a very high molecular mass (6-12 MDa) confer cancer resistance to naked mole rats and these Hyal have been shown to be refractory to endocytosis due to their larger size compared with those found in other organisms ${ }^{47}$. It is conceivable that such large biopolymers prohibit EMT by sequestering iron outside cells. Furthermore, although we have shown that Hyal can reversibly interact with iron, other metal ions can potentially be endocytosed using a similar glycosaminoglycan-mediated pathway and may also contribute to the regulation of epigenetic plasticity at different levels ${ }^{42}$. While our study illustrates a functional role of CD44, Hyal and iron in the context of tumorigenic cells, we anticipate that other physiological and pathological processes that rely on the status of distinct histone marks or modified DNA and RNAs are under the control of similar mechanisms that involve iron-dependent demethylases ${ }^{48-51}$.

\section{Online content}

Any methods, additional references, Nature Research reporting summaries, source data, extended data, supplementary information, acknowledgements, peer review information; details of author contributions and competing interests; and statements of data and code availability are available at https://doi.org/10.1038/ s41557-020-0513-5.

Received: 20 December 2019; Accepted: 16 June 2020;

Published online: 03 August 2020

\section{References}

1. Ponta, H., Sherman, L. \& Herrlich, P. A. CD44: from adhesion molecules to signalling regulators. Nat. Rev. Mol. Cell Biol. 4, 33-45 (2003).

2. Zöller, M. CD44: can a cancer-initiating cell profit from an abundantly expressed molecule? Nat. Rev. Cancer 11, 254-267 (2011).

3. Nieto, M. A., Huang, R. Y., Jackson, R. A. \& Thiery, J. P. EMT: 2016. Cell 166, 21-45 (2016)

4. Brabletz, T., Kalluri, R., Nieto, M. A. \& Weinberg, R. A. EMT in cancer. Nat. Rev. Cancer 18, 128-134 (2018).

5. Pastushenko, I. et al. Identification of the tumour transition states occurring during EMT. Nature 556, 463-468 (2018)

6. Günthert, U. et al. A new variant of glycoprotein CD44 confers metastatic potential to rat carcinoma cells. Cell 65, 13-24 (1991).

7. Aruffo, A., Stamenkovic, I., Melnick, M., Underhill, C. B. \& Seed, B. CD44 is the principal cell surface receptor for hyaluronate. Cell 61, 1303-1313 (1990).

8. Hua, Q., Knudson, C. B. \& Knudson, W. Internalization of hyaluronan by chondrocytes occurs via receptor-mediated endocytosis. J. Cell Sci. 106, 365-375 (1993).

9. Bartolazzi, A., Peach, R., Aruffo, A. \& Stamenkovic, I. Interaction between CD44 and hyaluronate is directly implicated in the regulation of tumor development. J. Exp. Med. 180, 53-66 (1994).

10. Zoltan-Jones, A., Huang, L., Ghatak, S. \& Toole, B. P. Elevated hyaluronan production induces mesenchymal and transformed properties in epithelial cells. J. Biol. Chem. 278, 45801-45810 (2003).

11. Toole, B. P. Hyaluronan: from extracellular glue to pericellular cue. Nat. Rev. Cancer 4, 528-539 (2004).

12. Mercê, A. L., Marques Carrera, L. C., Santos Romanholi, L. K. \& Lobo Recio, M. A. Aqueous and solid complexes of iron(III) with hyaluronic acid. Potentiometric titrations and infrared spectroscopy studies. J. Inorg. Biochem. 89, 212-218 (2002).

13. Torti, S. V. \& Torti, F. M. Iron and cancer: more ore to be mined. Nat. Rev. Cancer 13, 342-355 (2013).

14. Schonberg, D. L. et al. Preferential iron trafficking characterizes glioblastoma stem-like cells. Cancer Cell 28, 441-455 (2015).

15. Mai, T. T. et al. Salinomycin kills cancer stem cells by sequestering iron in lysosomes. Nat. Chem. 9, 1025-1033 (2017).

16. Basuli, D. et al. Iron addiction: a novel therapeutic target in ovarian cancer. Oncogene 36, 4089-4099 (2017).

17. Viswanathan, V. S. et al. Dependency of a therapy-resistant state of cancer cells on a lipid peroxidase pathway. Nature 547, 453-457 (2017).

18. Hangauer, M. J. et al. Drug-tolerant persister cancer cells are vulnerable to GPX4 inhibition. Nature 551, 247-250 (2017).

19. Morel, A. P. et al. Generation of breast cancer stem cells through epithelialmesenchymal transition. PLoS One 3, e2888 (2008).

20. Niwa, M., Hirayama, T., Okuda, K. \& Nagasawa, H. A new class of high-contrast $\mathrm{Fe}(\mathrm{II})$ selective fluorescent probes based on spirocyclized scaffolds for visualization of intracellular labile iron delivered by transferrin Org. Biomol. Chem. 12, 6590-6597 (2014).
21. Guerquin-Kern, J. L., Wu, T. D., Quintana, C. \& Croisy, A. Progress in analytical imaging of the cell by dynamic secondary ion mass spectrometry (SIMS microscopy). Biochim. Biophys. Acta 1724, 228-238 (2005).

22. Stuelten, C. H. et al. Complex display of putative tumor stem cell markers in the NCI60 tumor cell line panel. Stem Cells 28, 649-660 (2010).

23. Pantopoulos, K., Porwal, S. K., Tartakoff, A. \& Devireddy, L. Mechanisms of mammalian iron homeostasis. Biochemistry 51, 5705-5724 (2012).

24. Muckenthaler, M. U., Rivella, S., Hentze, M. W. \& Galy, B. A red carpet for iron metabolism. Cell 168, 344-361 (2017).

25. Chuang, C. H. et al. Molecular definition of a metastatic lung cancer state reveals a targetable CD109-Janus kinase-Stat axis. Nat. Med. 23, 291-300 (2017)

26. Bendris, N. et al. SNX9 promotes metastasis by enhancing cancer cell invasion via differential regulation of RhoGTPases. Mol. Biol. Cell 27, 1409-1419 (2016).

27. Feng, W., Yonezawa, M., Ye, J., Jenuwein, T. \& Grummt, I. PHF8 activates transcription of rRNA genes through $\mathrm{H} 3 \mathrm{~K} 4 \mathrm{me} 3$ binding and $\mathrm{H} 3 \mathrm{~K} 9 \mathrm{me} 1 / 2$ demethylation. Nat. Struct. Mol. Biol. 17, 445-450 (2010).

28. Fortschegger, K. et al. PHF8 targets histone methylation and RNA polymerase II to activate transcription. Mol. Cell. Biol. 30, 3286-3298 (2010).

29. Liu, W. et al. PHF8 mediates histone H4 lysine 20 demethylation events involved in cell cycle progression. Nature 466, 508-512 (2010).

30. Qi, H. H. et al. Histone H4K20/H3K9 demethylase PHF8 regulates zebrafish brain and craniofacial development. Nature 466, 503-507 (2010).

31. Shao, P. et al. Histone demethylase PHF8 promotes epithelial to mesenchymal transition and breast tumorigenesis. Nucleic Acids Res. 45, 1687-1702 (2017).

32. Carey, B. W., Finley, L. W., Cross, J. R., Allis, C. D. \& Thompson, C. B. Intracellular alpha-ketoglutarate maintains the pluripotency of embryonic stem cells. Nature 518, 413-416 (2015).

33. Bullock, G. C. et al. Iron control of erythroid development by a novel aconitase-associated regulatory pathway. Blood 116, 97-108 (2010).

34. Li, X., Egervari, G., Wang, Y., Berger, S. L. \& Lu, Z. Regulation of chromatin and gene expression by metabolic enzymes and metabolites. Nat. Rev. Mol. Cell Biol. 19, 563-578 (2018).

35. Smith, A. G., Carthew, P., Francis, J. E., Edwards, R. E. \& Dinsdale, D. Characterization and accumulation of ferritin in hepatocyte nuclei of mice with iron overload. Hepatology 12, 1399-1405 (1990).

36. Yu, L. et al. Structural insights into a novel histone demethylase PHF8. Cell Res. 20, 166-173 (2010).

37. McDonald, O. G., Wu, H., Timp, W., Doi, A. \& Feinberg, A. P. Genome-scale epigenetic reprogramming during epithelial-to-mesenchymal transition. Nat. Struct. Mol. Biol. 18, 867-874 (2011).

38. Zhao, Q. Y. et al. Global histone modification profiling reveals the epigenomic dynamics during malignant transformation in a four-stage breast cancer model. Clin. Epigenetics 8, 34 (2016).

39. McDonald, O. G. et al. Epigenomic reprogramming during pancreatic cancer progression links anabolic glucose metabolism to distant metastasis. Nat. Genet. 49, 367-376 (2017)

40. Gerry, C. J. \& Schreiber, S. L. Chemical probes and drug leads from advances in synthetic planning and methodology. Nat. Rev. Drug Discov. 17, 333-352 (2018)

41. Cañeque, T., Müller, S. \& Rodriguez, R. Visualizing biologically active small molecules in cells using click chemistry. Nat. Rev. Chem. 2, 202-215 (2018).

42. Müller, S. et al. Metformin reveals a mitochondrial copper addiction of mesenchymal cancer cells. PLoS One 13, e0206764 (2018).

43. Janzer, A. et al. Metformin and phenformin deplete tricarboxylic acid cycle and glycolytic intermediates during cell transformation and NTPs in cancer stem cells. Proc. Natl Acad. Sci. USA 111, 10574-10579 (2014).

44. Zilka, O. et al. On the mechanism of cytoprotection by ferrostatin-1 and liproxstatin-1 and the role of lipid peroxidation in ferroptotic cell death. ACS Cent. Sci. 3, 232-243 (2017).

45. Conrad, M. \& Pratt, D. A. The chemical basis of ferroptosis. Nat. Chem. Biol. 15, 1137-1147 (2019)

46. Flavahan, W. A., Gaskell, E. \& Bernstein, B. E. Epigenetic plasticity and the hallmarks of cancer. Science 357, eaal2380 (2017).

47. Tian, X. et al. High-molecular-mass hyaluronan mediates the cancer resistance of the naked mole rat. Nature 499, 346-349 (2013).

48. Gerken, T. et al. The obesity-associated FTO gene encodes a 2-oxoglutaratedependent nucleic acid demethylase. Science 318, 1469-1472 (2007).

49. Jia, G. et al. N6-methyladenosine in nuclear RNA is a major substrate of the obesity-associated FTO. Nat. Chem. Biol. 7, 885-887 (2011).

50. Greer, E. L. \& Shi, Y. Histone methylation: a dynamic mark in health, disease and inheritance. Nat. Rev. Genet. 13, 343-357 (2012).

51. Tsai, Y. P. et al. TET1 regulates hypoxia-induced epithelial-mesenchymal transition by acting as a co-activator. Genome Biol. 15, 513 (2014).

Publisher's note Springer Nature remains neutral with regard to jurisdictional claims in published maps and institutional affiliations.

(c) The Author(s), under exclusive licence to Springer Nature Limited 2020 


\section{Reporting summary}

Further information on research design is available in the Nature Research Reporting Summary linked to this article.

\section{Data availability}

All data are available in the manuscript or the Supplementary Information. Mass spectrometry data have been deposited at the ProteomeXchange Consortium (PRIDE Archive) with identifiers PXD011447 and PXD012862. ChIP-seq and RNA-seq data are available on the National Center for Biotechnology Information website with accession reference GSE121664. Source data are provided with this paper.

\section{Code availability}

Code employed for ChIP-seq and RNA-seq data analyses are available on Github at https://github.com/nservant/EMTiron.

\section{Acknowledgements}

We thank A. Puisieux, C. Hivroz and S. Dogniaux for providing us with HMLER and primary human T cells, the PICT-IBiSA@Pasteur Imaging Facility of Institut Curie, a member of the France-BioImaging national research infrastructure for the use of microscopes and SIMS, C. Gaillet for assistance with NMR spectroscopy, J.-L. Guerquin-Kern for assistance with SIMS sample preparation, the ICP-MS platform at the Institut de Physique du Globe de Paris, G. Arras for assistance with mass spectrometry data analysis, S. Durand and G. Kroemer for providing access to the metabolomics platform and P. Legoix for NGS sample preparation. The R.R. research group is funded by the European Research Council (ERC) under the European Union's Horizon 2020 research and innovation programme (grant agreement no. 647973), the Fondation Charles Defforey-Institut de France and Ligue Contre le Cancer (Equipe Labellisée). R.R. and D.L. are supported by Region IdF for NMR and MS infrastructures. The Institut de Physique du Globe de Paris is supported by the IPGP multidisciplinary program PARI and Paris-Region IdF (SESAME grant agreement no. 12015908). High-throughput sequencing was performed by the ICGex NGS platform of Institut Curie, supported by ANR-10-EQPX-03 (Equipex), ANR-10-INBS-09-08 (France Génomique Consortium) from the Agence Nationale de la Recherche (Investissements d'Avenir program) and by the Cancéropole IdF and the SiRIC-Curie program-SiRIC Grant (INCa-DGOS-4654).

\section{Author contributions}

R.R. conceptualized the study and directed the research. R.R., S.M. and F.S. designed the experiments. T.C. performed NMR spectroscopy and synthesized the clickable iron chelators. A.V. synthesized the iron(II)-specific fluorescent probes. S.M. produced knock out cell lines and performed the experiments in relation to iron endocytosis, which included western blotting, cell imaging, RNA interference, flow cytometry and ICP-MS. T.-D.W. performed SIMS imaging. A.L. performed RT-qPCR and subcellular fractionation experiments. F.S. prepared the samples for quantitative proteomics, metabolomics and next generation sequencing. B.L. and D.L. carried out quantitative proteomics. E.C.-J. and C.G. provided tumour samples and performed cell sorting. A.D., C.V. and S.B. provided assistance with the NGS library preparation. N.S. performed bioinformatics analysis. R.R., S.M. and F.S. interpreted the data and wrote the article.

\section{Competing interests}

R.R. is a founder, shareholder and serves on the scientific advisory board of SideROS.

\section{Additional information}

Extended data is available for this paper at https://doi.org/10.1038/s41557-020-0513-5. Supplementary information is available for this paper at https://doi.org/10.1038/ s41557-020-0513-5.

Correspondence and requests for materials should be addressed to R.R.

Reprints and permissions information is available at www.nature.com/reprints. 
a
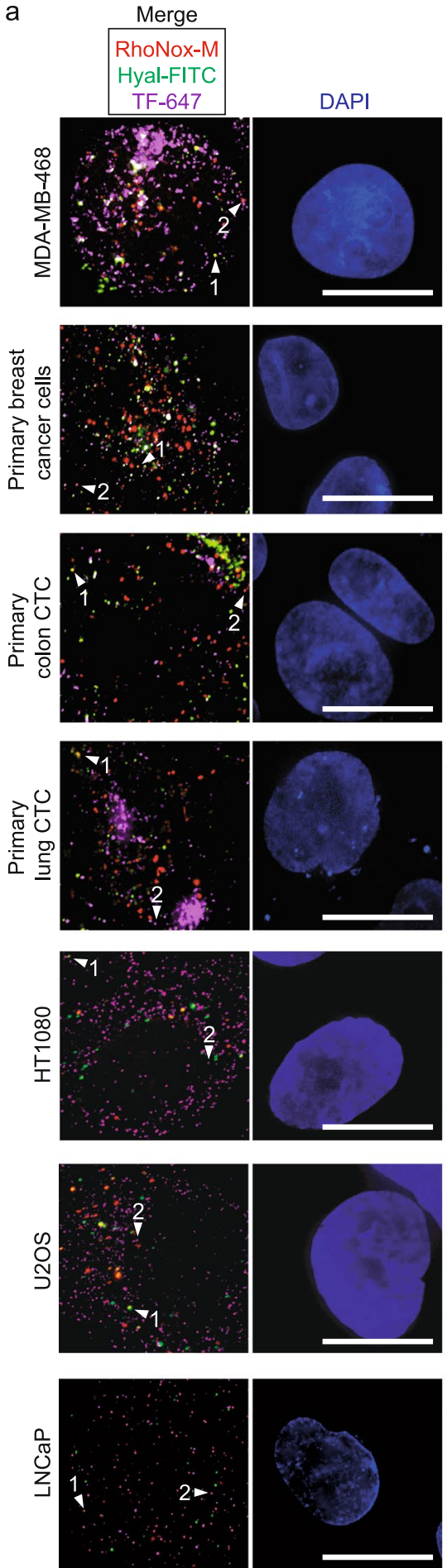

b

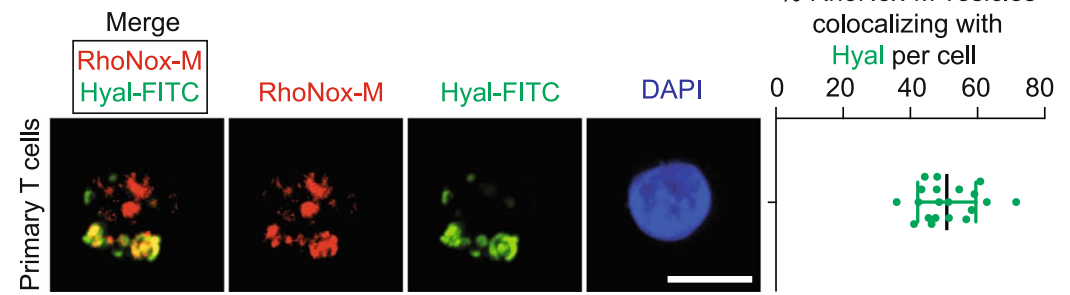

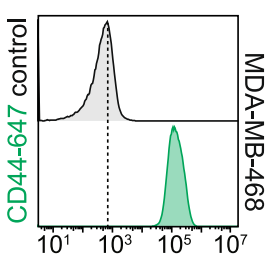
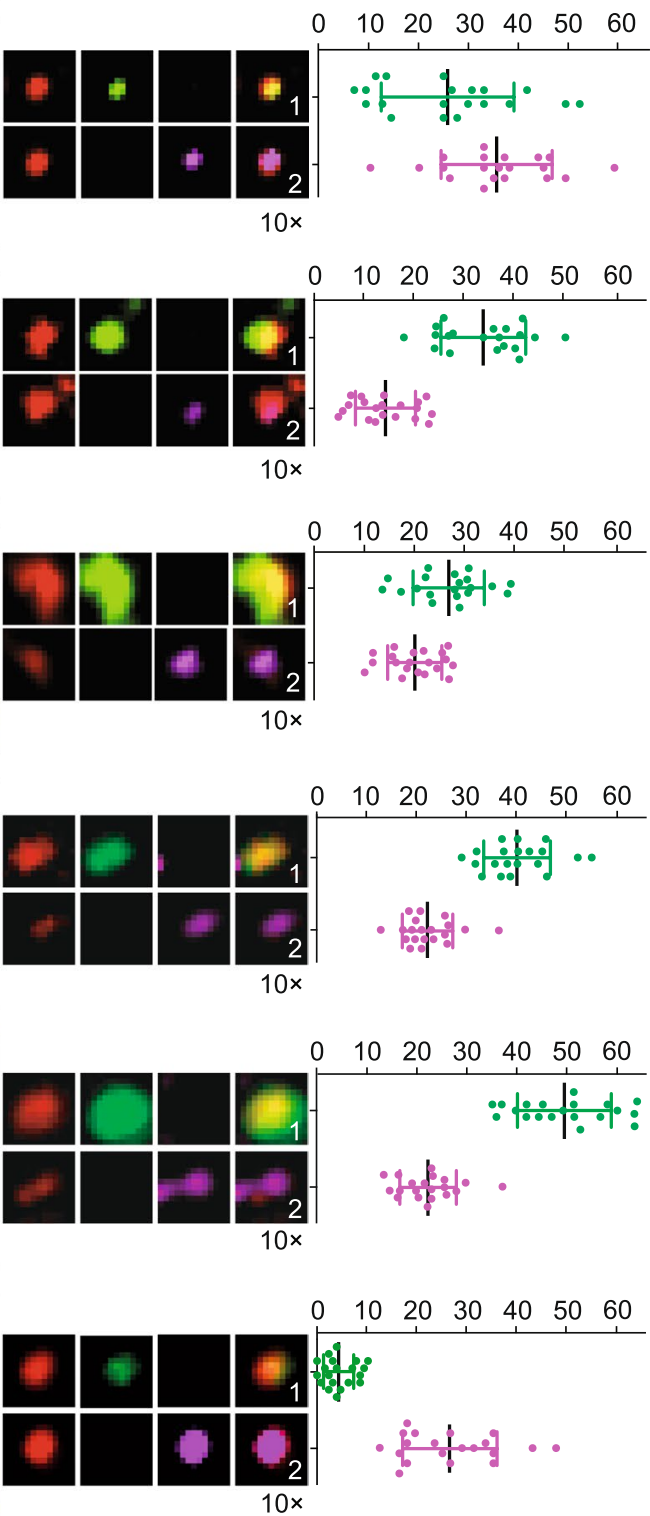

$\%$ RhoNox-M vesicles
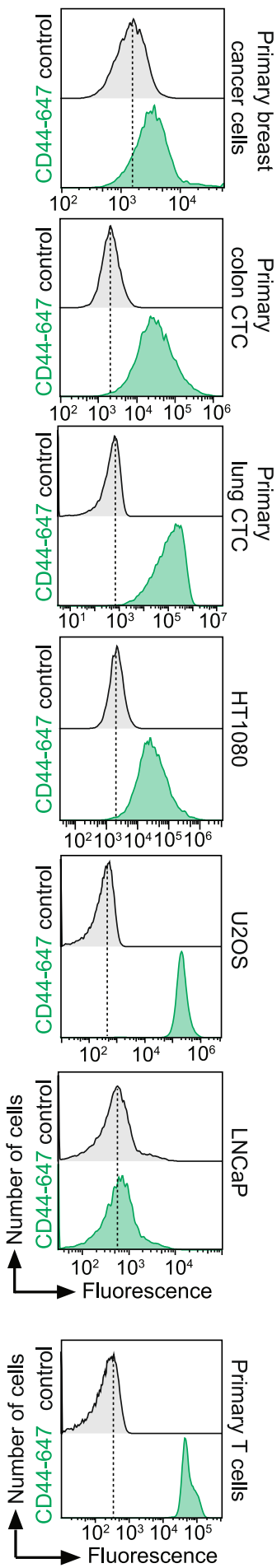

Extended Data Fig. 1 | See next page for caption. 
Extended Data Fig. 1 | CD44-mediates iron endocytosis in distinct cell lines and primary cells. a, Fluorescence microscopy of RhoNox-M-positive vesicles colocalizing with internalized Hyal-FITC or TF-647 (left panels). Scale bars, $10 \mu \mathrm{m} . n=3$ biologically independent experiments for MDA-MB-468, U2OS and HT1080 cell lines and $n=1$ for primary cancer cells and the LNCaP cell line. Flow cytometry of CD44 (right panels). b. Fluorescence microscopy of RhoNox-M-positive vesicles colocalizing with internalized Hyal-FITC in primary human $T$ cells (left panel). Scale bar, $2 \mu \mathrm{m} . n=3$ biologically independent experiments. Flow cytometry of CD44 (right panel). Bars and error bars, mean values \pm s.d. 
a

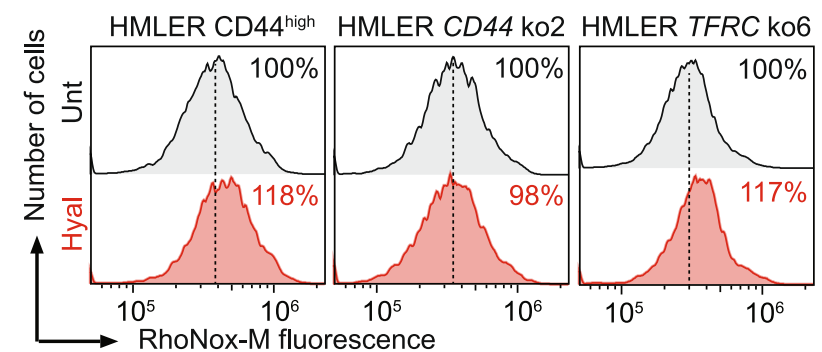

b

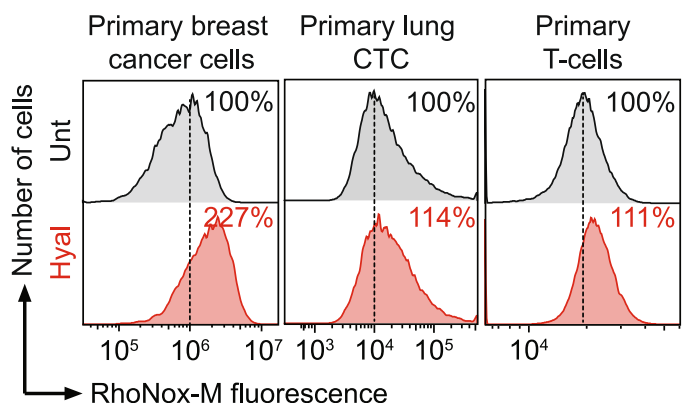

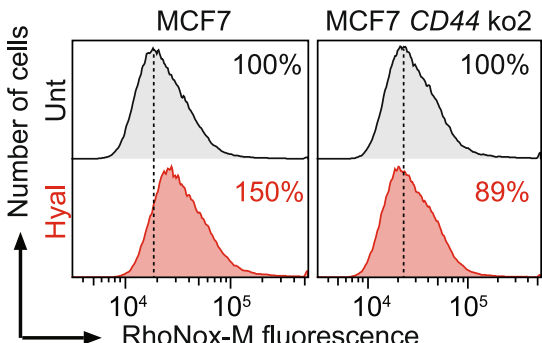

C

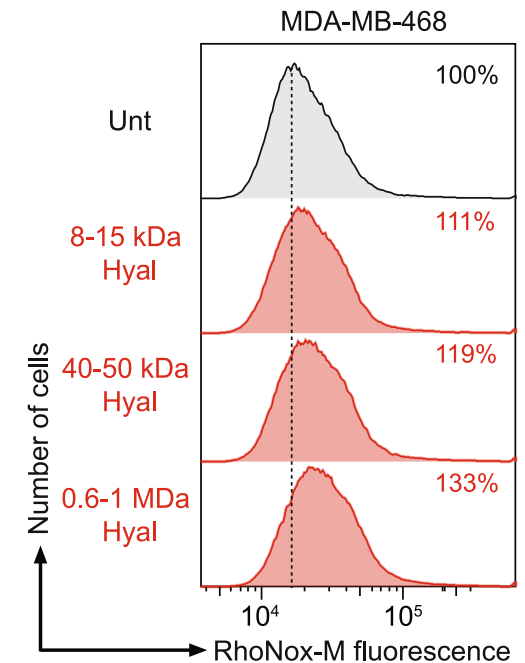

d

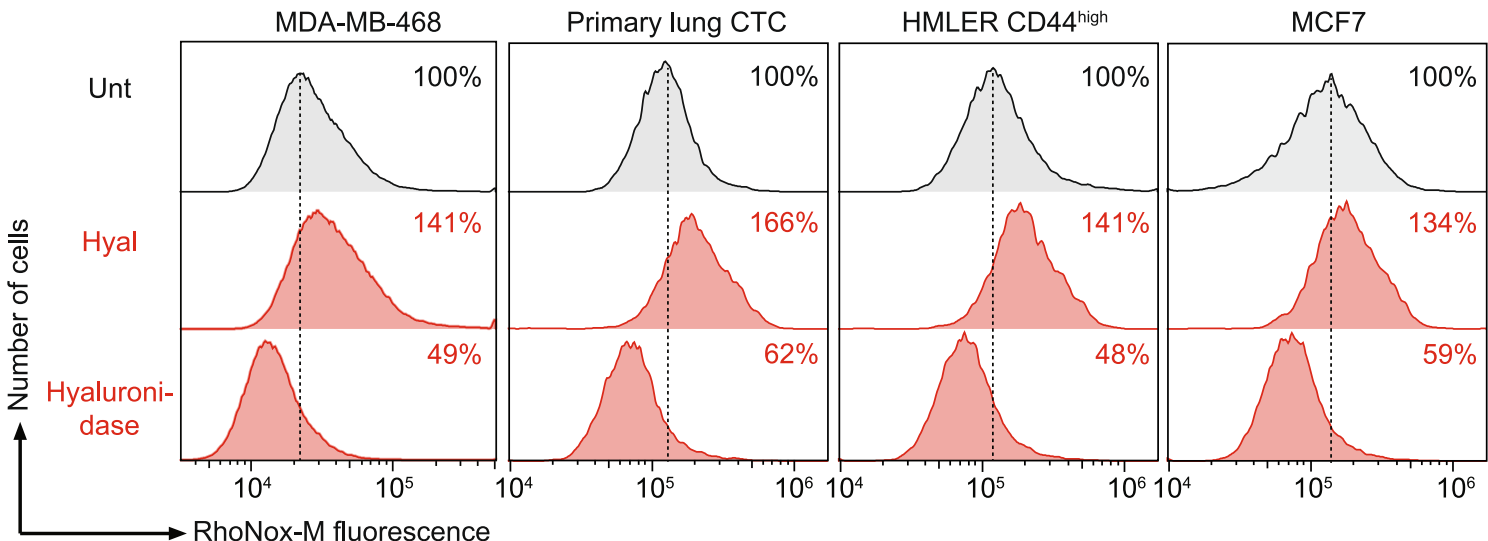

e

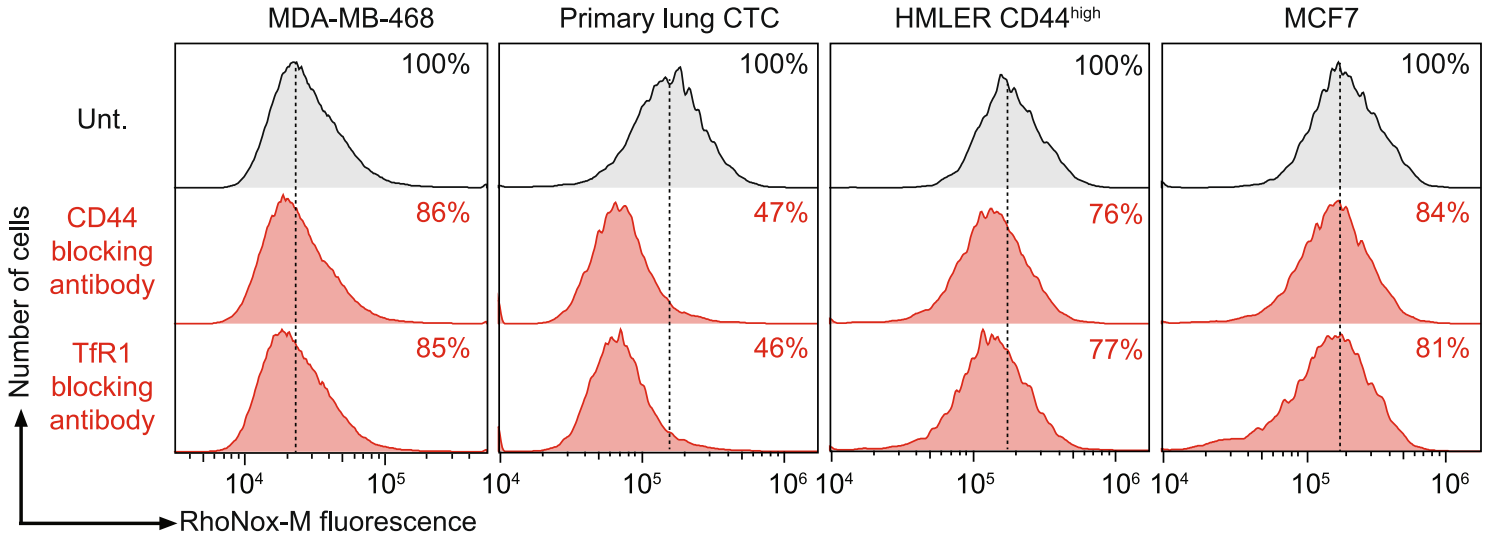

Extended Data Fig. 2 | CD44-mediated iron uptake is Hyal-dependent in distinct cell lines and primary cells. a, Flow cytometry of RhoNox-M fluorescence in HMLER CD44 and TFRC ko clones and a MCF7 CD44 ko clone treated with Hyal. b, Flow cytometry of RhoNox-M fluorescence in primary cancer cells and primary T cells treated with Hyal. $n=1$. c, Flow cytometry of RhoNox-M fluorescence in MDA-MB-468 cells treated with Hyal of varying molecular mass. $\mathbf{d}$, and e, Flow cytometry of RhoNox-M in MDA-MB-468, primary lung CTC, HMLER CD44high and MCF7 cells treated with Hyal,

hyaluronidase or blocking antibodies. $n=1$. HMM Hyal (0.6-1 MDa) was used in $\mathbf{a}, \mathbf{b}, \mathbf{d}$. Data representative of $n=3$ biologically independent experiments for $\mathbf{a}$ and $\mathbf{c}$. 
a

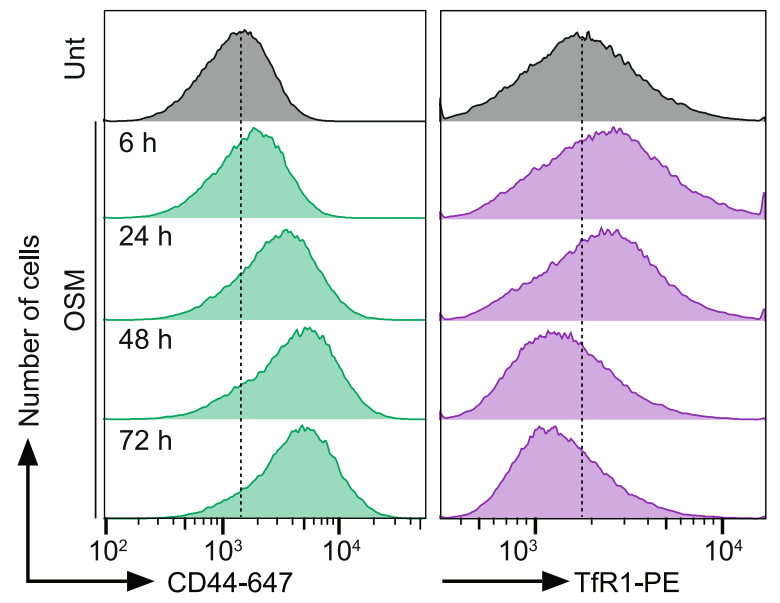

MCF7
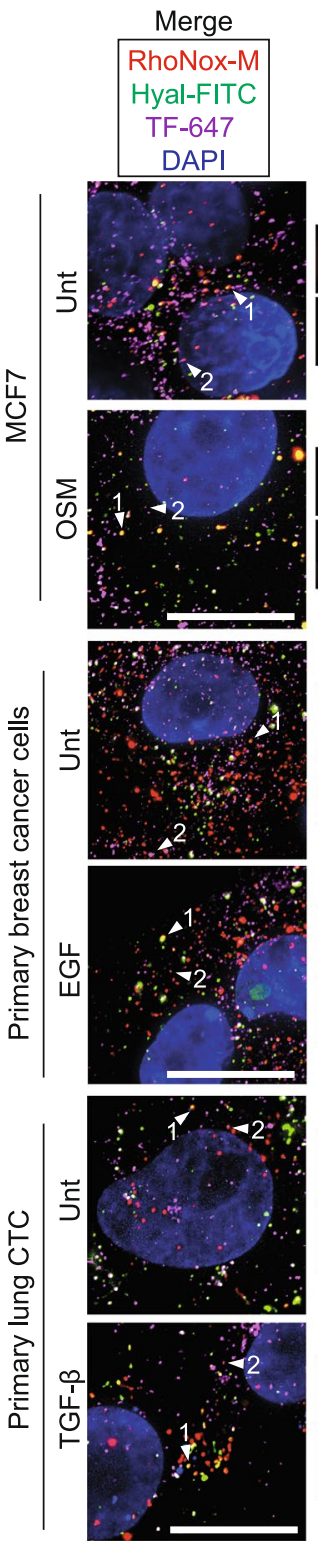

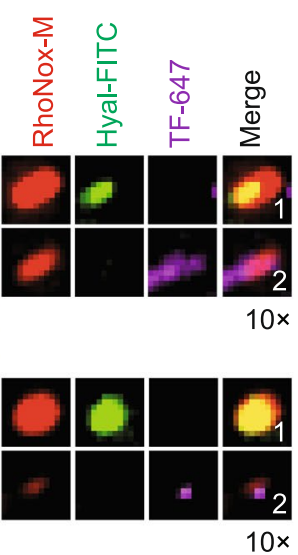

$\%$ RhoNox-M vesicles

colocalizing with

Hyal or TF per cell

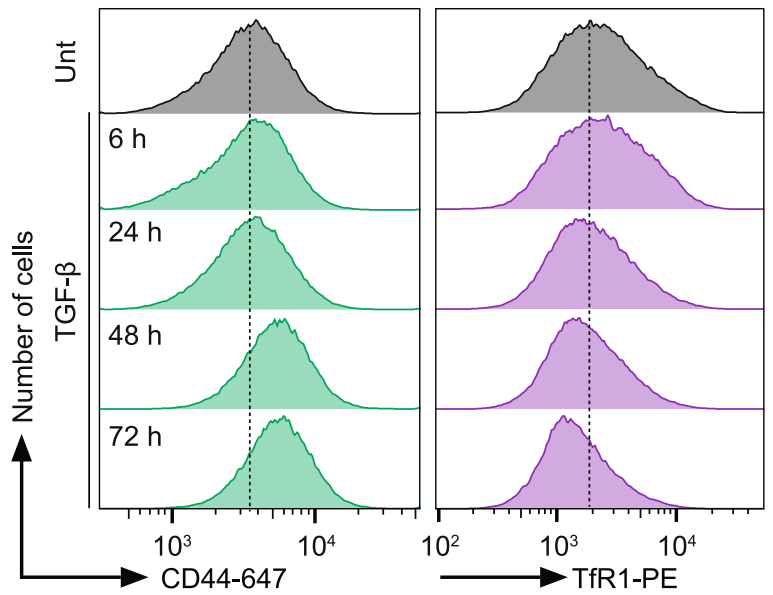

b
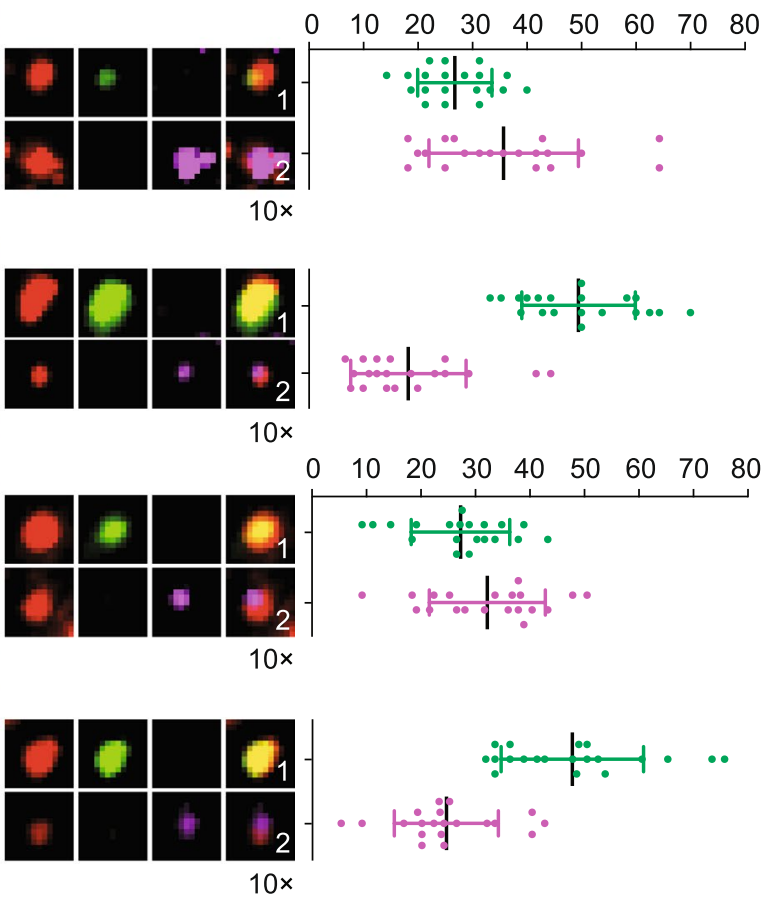

Extended Data Fig. 3 | CD44-mediated iron endocytosis prevails in the mesenchymal state of cells. a, Time course flow cytometry of CD44 and TfR1 at plasma membrane of MCF7 and HMLER CD44 ${ }^{\text {low }}$ cells treated with OSM or TGF- $\beta$ as indicated. Data representative of $n=3$ biologically independent experiments. b, Fluorescence microscopy of RhoNox-M-positive vesicles colocalizing with internalized Hyal-FITC or TF-647 in cells treated as indicated for $72 \mathrm{~h}$. Scale bars, $10 \mu \mathrm{m} . n=3$ biologically independent experiments for MCF7 cells and $n=1$ for primary cells. Bars and error bars, mean values \pm s.d. Unpaired t-tests, two-sided. 
a
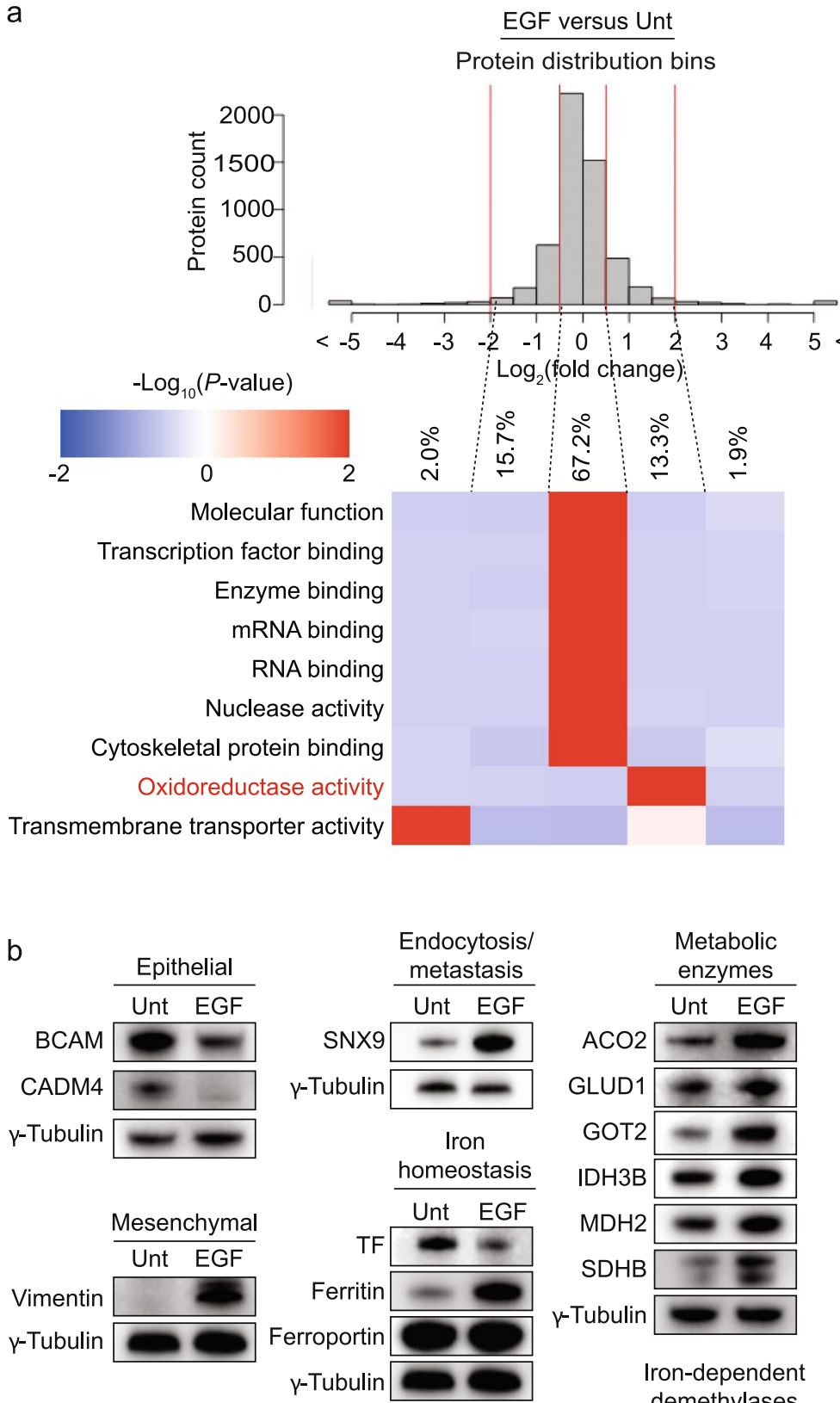

Vimentin Y-Tubulin

Mesenchymal Unt EGF

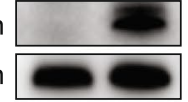
-Tubulin

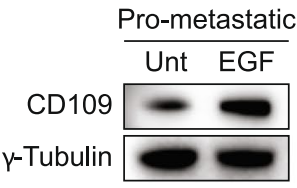

ron-dependent demethylases

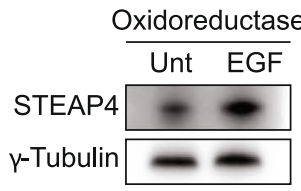

PHF8 Unt EGF

N066

KDM3B

$\mathrm{KDM} 4 \mathrm{C}$
C

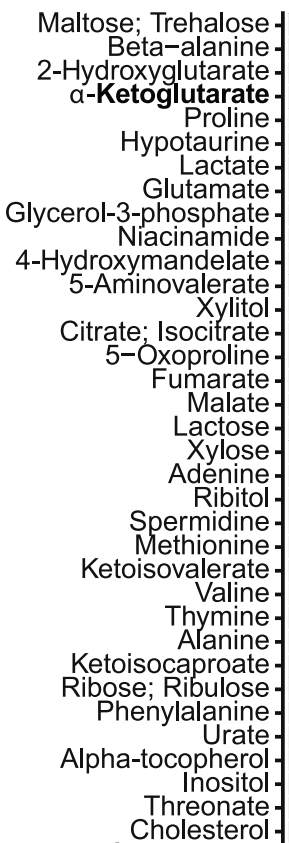
Pyruvate; Oxaloacetate 4-Hydroxyproline Erythritol.

Galactitol; Sorbitol: Mannitol Phosphate Arabitol. Glycerate ThreonineGlycine.

Phosphoenolpyruvate Isoleucine$\mathrm{N}$-acetylputrescine 3-Hydroxybutyrate. Shikimate. Ornithine-

Pantothenate Succinate Margarate Stearate Laurate. Palmitate Cytosine Myristate Aspartate LysineUracil Leucine

Y-Tubulin

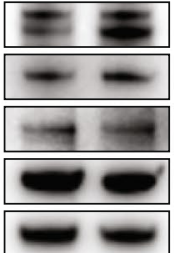

Homoserine
2-Aminoadipate

O-phosphoethan Linolate

Asparagine

Fructose -

Histidine

Galactose; Glucose; Mannose Citrulline-
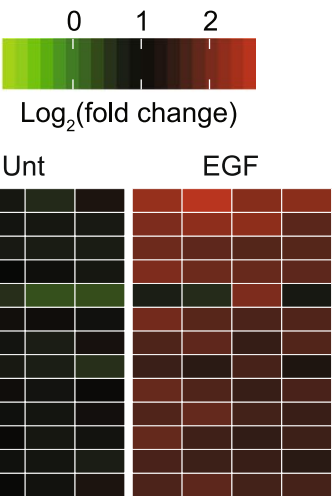

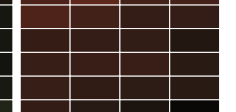

$+0$

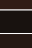

\begin{tabular}{|l}
\hline \\
\hline \\
\hline
\end{tabular}

\begin{tabular}{l|}
\hline \\
\hline \\
\hline+ \\
\hline+ \\
\hline+
\end{tabular}
Serine-

Arabinose

Sebacate -

Oleate: Elaidate -6-phosphate Arachidonate

Palmitoleate

Inosine -

Xanthine

Creatine -

Putrescine -

Tryptophan

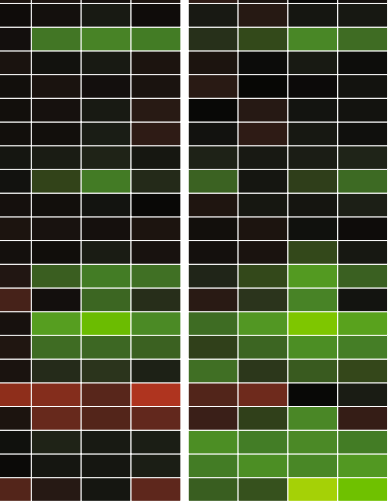

Extended Data Fig. 4 | See next page for caption. 
Extended Data Fig. 4 | Quantitative analysis of proteins and metabolites in cells undergoing EMT. a, Gene Ontology-term enrichment heatmap of proteins ranked by molecular function illustrating a bias towards proteins with oxidoreductase activity being increased in the mesenchymal cell state. $n=3$ biologically independent experiments. Statistical analysis, Material and Methods. $\mathbf{b}$, Western blots of selected proteins. Data representative of $n=3$ biologically independent experiments. c, Heatmap of metabolites in cells treated with EGF for $60 \mathrm{~h} . n=4$ technical replicates. MDA-MB-468 cells throughout the figure and treated with EGF for $72 \mathrm{~h}$ unless stated otherwise. 
a

H3K9me2 ChIP-seq

$\frac{10 \mathrm{~kb}}{V I M}$

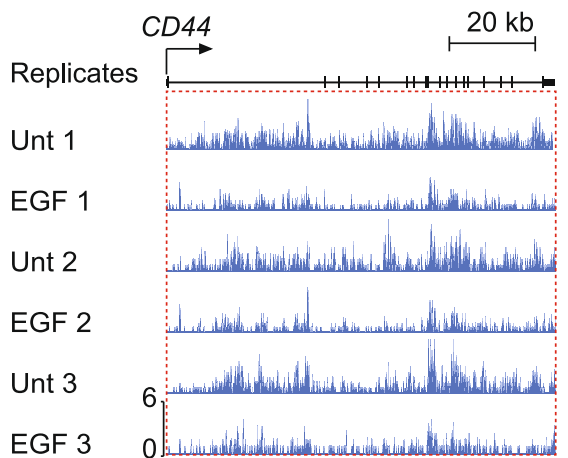
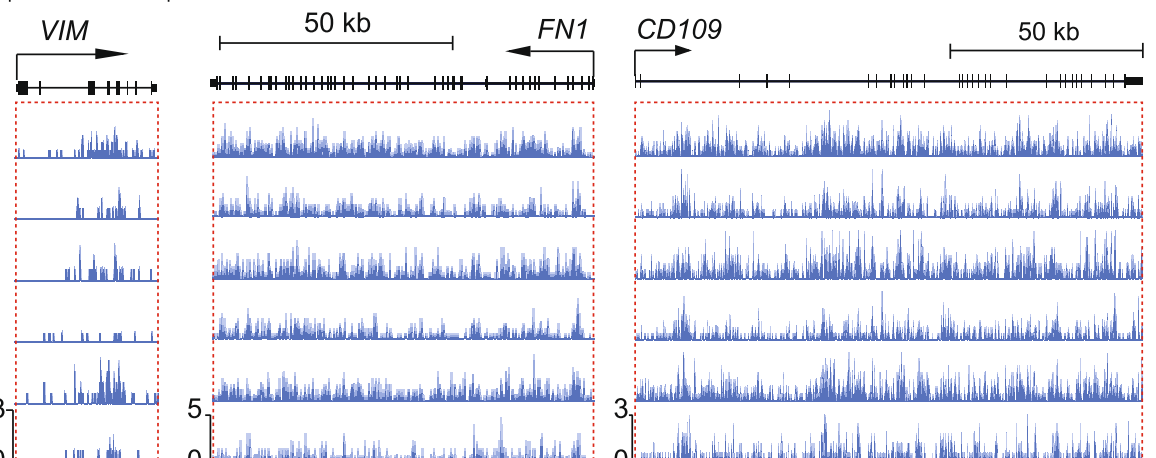

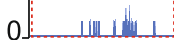

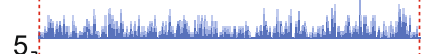

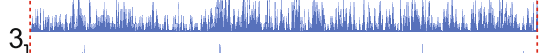

b H3K9me2 ChIP-qPCR
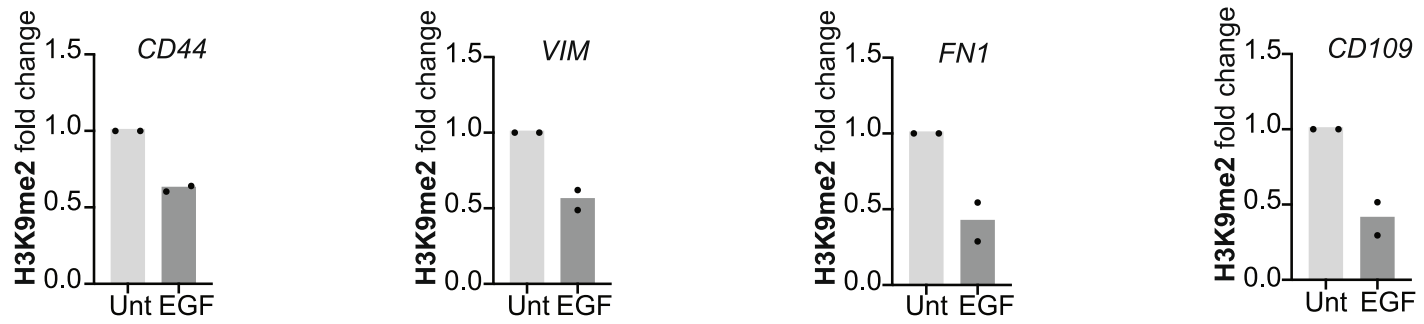

C H3K4me3 ChIP-seq

$10 \mathrm{~kb}$
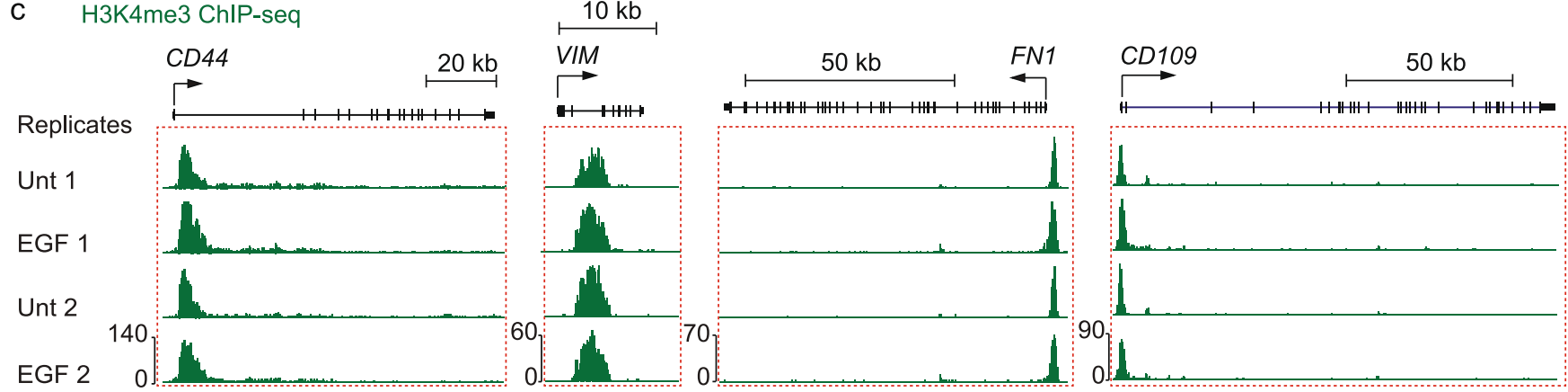

d H3K27me3 ChIP-seq
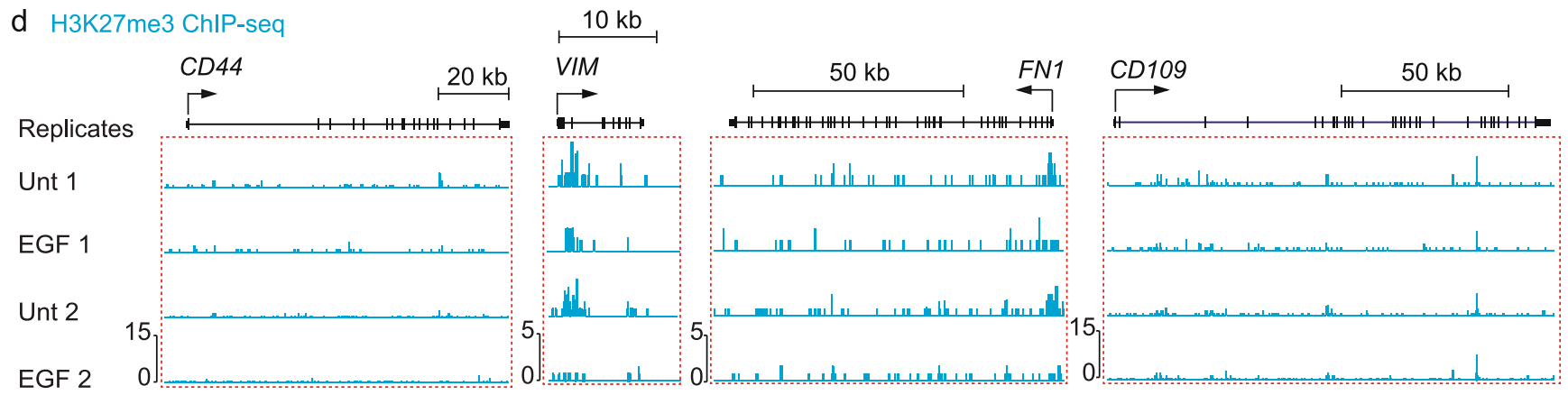

e H3K9me3 ChIP-seq

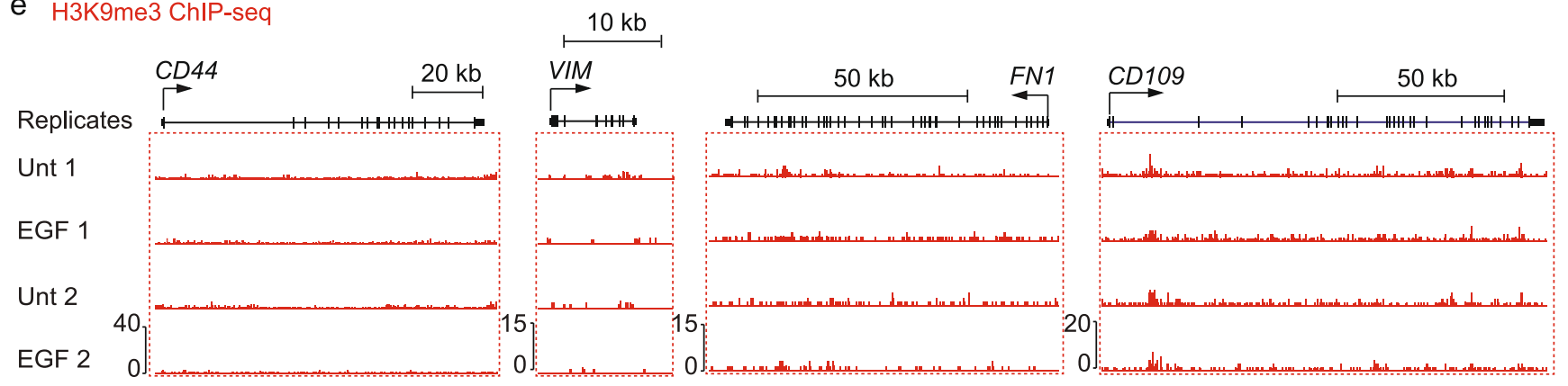

Extended Data Fig. 5 | Genome-wide analysis of histone marks in cells undergoing EMT. a, H3K9me2 ChIP-seq profiles for selected genes. $n=3$ biologically independent experiments. $\mathbf{b}, \mathrm{ChIP-qPCR}$ of selected genes. $n=2$ biologically independent experiments. c-e, ChIP-seq profiles of H3K 4 me3, H3K27me3 and H3K9me3 for selected genes. $n=2$ biologically independent experiments. MDA-MB-468 cells were used throughout the figure and treated with EGF for $72 \mathrm{~h}$. 
a
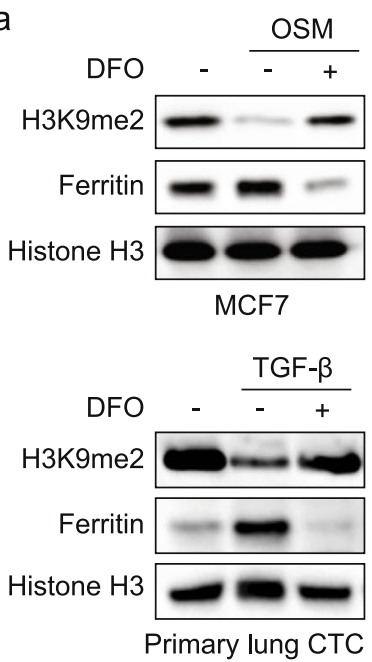

c

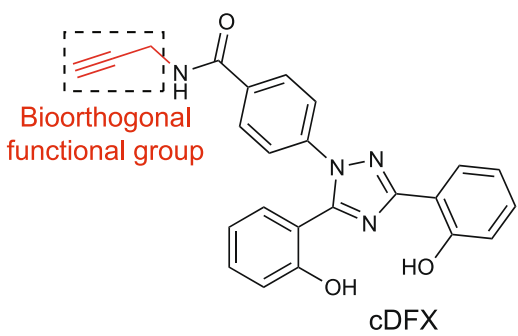

MDA-MB-468

d

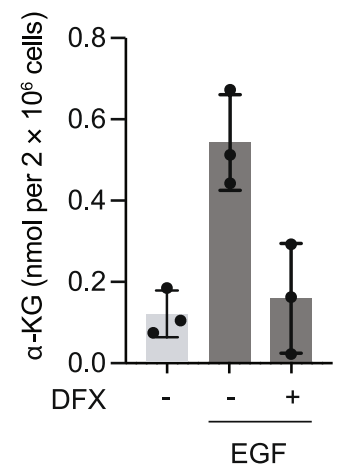

b
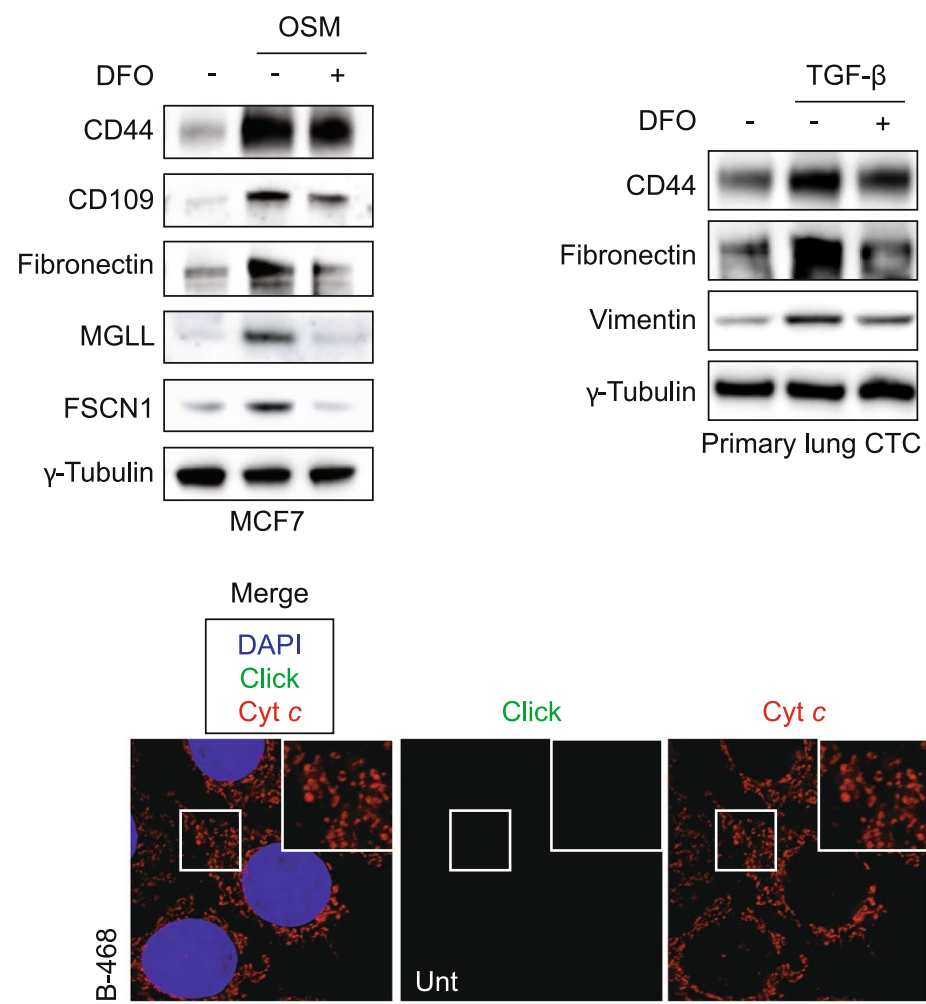

Cyt $c$
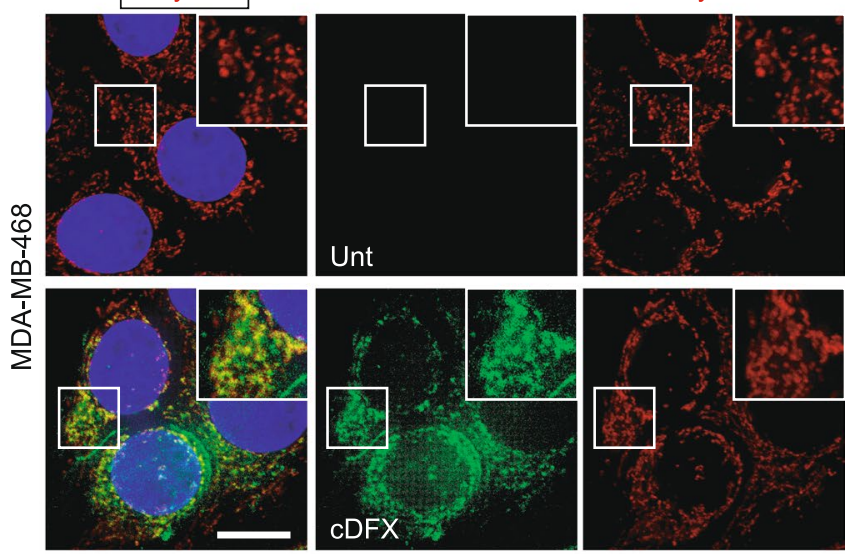

e

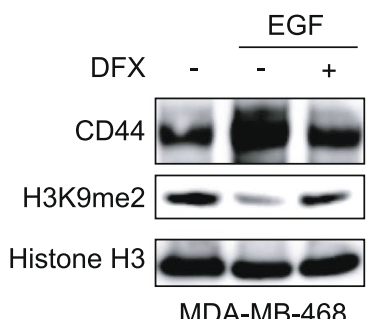

Extended Data Fig. 6 | Pharmacological targeting of iron-regulated processes. a, Western blots of H3K9me2 in cells co-treated with OSM and DFO or TGF- $\beta$ and DFO as indicated. $n=1$ for primary cells. $\mathbf{b}$, Western blots of proteins whose genes are regulated by H3K9me2 in cells co-treated with OSM and DFO or TGF- $\beta$ and DFO as indicated. $n=1$ for primary cells. $\boldsymbol{c}$, Molecular structure of clickable deferasirox (CDFX) (left), fluorescence microscopy of labelled cDFX and the mitochondrial component Cyt $c$ (right). Scale bar, $10 \mu \mathrm{m}$. d, $\alpha \mathrm{KG}$ quantification assay of MDA-MB-468 cells co-treated with EGF and deferasirox (DFX). $n=3$ technical replicates. e, Western blots of CD44 and H3K9me2 in MDA-MB-468 cells co-treated with EGF and DFX. Data representative of $n=3$ biologically independent experiments throughout the figure unless stated otherwise. Bars and error bars, mean values \pm s.d. 


\section{natureresearch}

Corresponding author(s): Raphaël Rodriguez

Last updated by author(s): 13 May 2020

\section{Reporting Summary}

Nature Research wishes to improve the reproducibility of the work that we publish. This form provides structure for consistency and transparency in reporting. For further information on Nature Research policies, see Authors \& Referees and the Editorial Policy Checklist.

\section{Statistics}

For all statistical analyses, confirm that the following items are present in the figure legend, table legend, main text, or Methods section.

n/a Confirmed

$\bigotimes$ The exact sample size $(n)$ for each experimental group/condition, given as a discrete number and unit of measurement

$\square \bigotimes$ A statement on whether measurements were taken from distinct samples or whether the same sample was measured repeatedly

The statistical test(s) used AND whether they are one- or two-sided

$\square$ Only common tests should be described solely by name; describe more complex techniques in the Methods section.

Х $\square$ A description of all covariates tested

Х $\square$ A description of any assumptions or corrections, such as tests of normality and adjustment for multiple comparisons

$\triangle$ A full description of the statistical parameters including central tendency (e.g. means) or other basic estimates (e.g. regression coefficient)

AND variation (e.g. standard deviation) or associated estimates of uncertainty (e.g. confidence intervals)

X For null hypothesis testing, the test statistic (e.g. $F, t, r$ ) with confidence intervals, effect sizes, degrees of freedom and $P$ value noted

$\square$ Give $P$ values as exact values whenever suitable.

\ $\square$ For Bayesian analysis, information on the choice of priors and Markov chain Monte Carlo settings

Х $\square$ For hierarchical and complex designs, identification of the appropriate level for tests and full reporting of outcomes

$\bigotimes \square$ Estimates of effect sizes (e.g. Cohen's $d$, Pearson's $r$ ), indicating how they were calculated

Our web collection on statistics for biologists contains articles on many of the points above.

\section{Software and code}

Policy information about availability of computer code

Data collection Microscopy data were collected using SoftWorx. Flow Cytometry data were recorded using Cell Quest (BD Biosciences). RT-PCR data were recorded using Light Cycler 480 (Roche) software. SIMS data were recorded with the software of a NanoSIMS-50 ion microprobe (Cameca, Gennevilliers, France). ICP-MS data were recorded using the software of a Agilent 7900 ICP-QMS. Metabolomics data were detected and integrated using the Agilent Mass Hunter quantitative software.

Data analysis

Microscopy data were deconvoluted using SoftWorx and analysed with Image J software. SIMS data were analysed using ImageJ. Data were plotted and statistics analysed using Prism 7. Flow cytometry data were analysed with FloJo software. Mass spectrometry data were analysed using myProMS software. ChIP-Seq and RNA-Seq data were analysed as described in the ChIP-Seq section and methods.

For manuscripts utilizing custom algorithms or software that are central to the research but not yet described in published literature, software must be made available to editors/reviewers. We strongly encourage code deposition in a community repository (e.g. GitHub). See the Nature Research guidelines for submitting code \& software for further information.

\section{Data}

Policy information about availability of data

All manuscripts must include a data availability statement. This statement should provide the following information, where applicable:

- Accession codes, unique identifiers, or web links for publicly available datasets

- A list of figures that have associated raw data

- A description of any restrictions on data availability

All data and codes are available in the manuscript or the supplementary materials. Mass spectrometry data have been deposited at the ProteomeXchange Consortium (PRIDE Archive) with identifiers PXD011447 and PXD012862. ChIP-seq and RNA-seq data are available on the National Center for Biotechnology Information website with accession reference GSE121664. Code employed for ChIP-seq and RNA-seq data analyses are available on Github at https://github.com/ nservant/EMTiron. 


\section{Field-specific reporting}

Please select the one below that is the best fit for your research. If you are not sure, read the appropriate sections before making your selection. $\bigotimes$ Life sciences $\quad \square$ Behavioural \& social sciences $\quad \square$ Ecological, evolutionary \& environmental sciences

For a reference copy of the document with all sections, see nature.com/documents/nr-reporting-summary-flat.pdf

\section{Life sciences study design}

All studies must disclose on these points even when the disclosure is negative.

Sample size We have used at least three independent biological replicates unless stated otherwise. For experiments using individual cells we quantified the number as indicated in the manuscript, generally at least 20. This is consistent with previous studies and accounts for biological variability between samples. No statistical method was used to predetermine sample size.

Data exclusions There are no data excluded from this study.

Replication Experimental findings were reliably reproduced.

Randomization Fluorescence microscopy images were selected randomly using the DAPI channel in each group for quantifications. Cells were chosen randomly in each group for SIMS imaging and multiframe acquisition was applied, taking sequential measurements of treated and untreated samples.

Blinding Experiments were blinded for the SIMS and ICP-MS experiments.

\section{Reporting for specific materials, systems and methods}

We require information from authors about some types of materials, experimental systems and methods used in many studies. Here, indicate whether each material, system or method listed is relevant to your study. If you are not sure if a list item applies to your research, read the appropriate section before selecting a response.

\begin{tabular}{l|l|l} 
Materials \& experimental sys \\
\hline $\mathrm{n} / \mathrm{a}$ & Involved in the study \\
\hline & $\bigotimes$ Antibodies \\
$\square$ & $\square$ Animals and other organisms \\
$\square$ & $\square$ Human research participants \\
$\square$ & $\square$ Clinical data
\end{tabular}

\begin{tabular}{l|l}
\multicolumn{2}{l}{ Methods } \\
\hline n/a & Involved in the study \\
$\square$ & $\bigotimes$ ChIP-seq \\
$\square$ & $\square$ Flow cytometry \\
\hline
\end{tabular}

\section{Antibodies}

Antibodies used

(WB = Western blot, FACS = Fluorescence-activated cell sorting, FC = Flow cytometry, FM = Fluorescence microscopy). ACO2 (Cell Signaling, 6922S, WB: 1:1000), BCAM (ThermoFisher Scientific, PA5-50376, WB: 1:1000), CADM-4 (Abcam, ab150370, WB: 1:1000), CD109 (Santa Cruz Biotechnology, sc-271085, WB: 1:500), CD24-APC (Sony Biotechnology Inc., 2155590, FACS: $1 \mu \mathrm{L} / 106$ cells), CD44 (Abcam, ab189524, WB: 1:30000, FM of permeabilized cells: 1:200), CD44 (Abcam, ab25340, blocking: $10 \mathrm{~g} / \mathrm{mL}, 1 \mathrm{~h}$ ), CD44 Alexa-Fluor-647 (Novus Biologicals, NB500-481AF647, FC), CD44-PE (R\&D Systems, FAB4948P, FACS: $10 \mu \mathrm{L} / 106$ cells), CDCP1 (Cell Signaling, 4115, WB: 1:1000), Cytochrome c (Cyt c, Cell Signaling, 12963, IF 1:500), Drosophila spike-in antibody (Active Motif, 61686, ChIP-seq: 1 g), E-cadherin (Cell Signaling, 3195, WB: 1:1000, IF: 1:200), Ferritin (Abcam, ab75973, WB: 1:1000), Ferroportin (Novus Biologicals, \#NBP1-21502SS, WB: 1:1000), Fibronectin (Sigma-Aldrich, F0791, WB: 1:1000), FSCN1 (Abcam, ab126772, WB: 1:1000), GLUD1 (Abcam, ab166618, WB: 1:5000), GOT2 (Abcam, ab184012, WB: 1:10000), H3 (Cell Signaling, 9715S, WB: 1:106), H3K4me3 (Diagenode, C15410003-50, ChIP-seq: 3 L), H3K9me2 (Cell Signaling, 4658S, WB: 1:1000, ChIP-seq: 6 L), H3K9me3 (Cell Signaling, 13969S, ChIP-seq: 6 L), H3K27me3 (Cell Signaling, 9733S, ChIP-seq: 6 L), IDH3B (Abcam, ab112544, WB: 1:1000), KDM3B (Cell Signaling, 2621S, WB: 1:1000), KDM4C (Abcam, ab85454, WB: 1:1000), MDH2 (Abcam, ab96193, WB: 1:1000), MGLL (Abcam, ab24701, WB: 1:1000), NO66 (Cell Signaling, 20779S, WB: 1:1000), PHF8 (Active Motif, 39711, WB: 1:1000), SDHB (Abcam, ab178423, WB: 1:5000), Snail (Cell Signaling, 3895, WB: 1:1000), SNX9 (Proteintech, 15721-1-AP, WB:1:1000), STEAP4 (Proteintech, 11944-1-AP, WB: 1:1000), Transferrin (Tf, Abcam, ab137744, WB:1:1000), Transferrin receptor 1 (TfR1, Life Technologies, 13-6800, WB: 1:1000), Transferrin receptor 1 (TfR1, Merck Millipore, GR09L-100UG, blocking: $10 \mathrm{~g} / \mathrm{mL}, 1 \mathrm{~h}$ ), TfR1-PE (R\&D Systems, FAB2474P, FC: 5 pL/106 cells), $\beta$-Tubulin (Sigma-Aldrich, T4026-100UL, WB: 1:2000), $\psi$-Tubulin (Sigma-Aldrich, T5326, WB: 1:2000), Vimentin (Cell Signaling, 5741S, WB: 1:500), Zeb1 (Santa Cruz Biotechnology, sc-81428, WB: 1:500). Secondary antibodies for WB: HRP anti-Mouse (Bethyl Laboratories, A90-116P, 1:10000) and HRP anti-Rabbit (Bethyl Laboratories, A120-108P, 1:10000). Secondary antibodies for fluorescence microscopy: Alexa-Fluor-488 conjugate (Life Technologies, A-11017 Mouse, A-11008 Rabbit, 1:1000), Alexa-Fluor-594 conjugate (Life 
Technologies, A-11032 Mouse, A-11072 Rabbit, 1:1000), Alexa-Fluor-647 conjugate (Life Technologies, A-20990 Mouse, A-20991 Rabbit, 1:500).

\section{Eukaryotic cell lines}

\section{Policy information about cell lines}

Cell line source(s)

MDA-MB-468 (ATCC, HTB-132, sex: female), HT1080 (ATCC, CCL-121, sex: male), U2OS (ATCC, HTB-96, sex: female) and MCF7 (ATCC, HTB-22, sex: female) cells and MCF7 CD44 ko clones were cultured in Dulbecco's Modified Eagle Medium GlutaMAX (DMEM, ThermoFisher Scientific, 61965059) supplemented with 10\% Fetal Bovine Serum (FBS, Gibco, 10270-106) and Penicillin-Streptomycin mixture (BioWhittaker/Lonza, DE17-602E) unless stated otherwise. LNCaP (ATCC, CRL-1740, sex: male) cells were cultured in Roswell Park Memorial Institute medium GlutaMAX (RPMI-1640, ThermoFisher Scientific, 61870044) supplemented with 10\% Fetal Bovine Serum (FBS, Gibco, 10270-106) and Penicillin-Streptomycin mixture (BioWhittaker/Lonza, DE17-602E). HMLER cells (sex: female) naturally repressing E-cadherin, obtained from human mammary epithelial cells infected with a retrovirus carrying hTERT, SV4O and the oncogenic allele H-rasV12, and HMLER CD44 and TFRC ko clones were cultured in DMEM/F12 (Gibco, 31331-028) supplemented with 10\% FBS, $10 \mu \mathrm{g} / \mathrm{mL}$ insulin (Sigma-Aldrich, 10516), $0.5 \mu \mathrm{g} / \mathrm{mL}$ hydrocortisone (Sigma-Aldrich, H0888) and $0.5 \mu \mathrm{g} / \mathrm{mL}$ puromycin (Life Technologies, A11138-02), unless stated otherwise. HMLER CD44high cells were also supplemented with $10 \mathrm{ng} / \mathrm{mL}$ EGF, whereas HMLER CD44low cells were grown without EGF. HMLER CD44low/high cells stained with CD24-APC and CD44-PE antibodies were sorted by FACS using an Aria Ilu (BD Biosciences) to obtain isolated CD44high/CD24low and CD44low/CD24high cell populations. Primary breast cancer cells (Celther Polska, RT-04002-CLTH, Lot 074325, sex: female) were grown in BCFM medium (Celther Polska, MED 02002-CLTH) and kept in culture until the third passage. Primary lung circulating tumor cells (Celprogen, 36107-34CTC, Lot 219411, sex: female) and primary colon circulating tumor cells (Celprogen, 36112-39CTC, Lot 20188, sex: female) were grown using stem cell complete media (Celprogen, M36102-29PS) until the third passage. Circulating cancer cells were grown in stem cell ECM T75-flasks (Celprogen, E36102-29-T75) and ECM 6-well plates (Celprogen, E36102-29-6Well). Primary human T-cells were cultured in RPMI 1640 medium (Gibco, 61870-010) supplemented with $10 \%$ FBS. All cells were incubated at $37 \mathrm{C}$ with $5 \% \mathrm{CO} 2$.

\section{Authentication}

No methods were used for cell line authentication.

Mycoplasma contamination

Commonly misidentified lines (See ICLAC register)
All cell lines were tested negative for mycoplasma contamination.

No commonly misidentified cell lines were used in this study.

\section{Human research participants}

Policy information about studies involving human research participants

Population characteristics Cells from two primary breast tumors were implanted in immunodeficient mice as previously described (Charafe-Jauffret et al., 2013), generating a human-into-mice model of aggressive HER2 (CRCM 226) and triple negative (CRCM 330) breast cancers. These two models, which gave rise to metastasis in patients (skin and lung for CRCM 226; lung, node and brain for CRCM 330), were established respectively prior and after taxane- and anthracyclin-based chemotherapy. Tumors were grown in mice for 46 and 33 days (6th and 7th passages into animals). Tumor samples were freshly collected from mice in triplicate for each PDX model and dissociated in single cell suspensions, which were incubated with human CD44-APC (BD Biosciences, 559942, FC: 100 $\mu \mathrm{L} / 106$ cells) and murine $\mathrm{H}-2 \mathrm{kd}-\mathrm{FITC}$ (BD Biosciences, 553565, FC: $100 \mu \mathrm{L} / 106$ cells) antibodies. CD44high and CD44low human cancer cells (negative for $\mathrm{H}-2 \mathrm{kd}$ ) were then sorted after viability selection (eBioscience FVD eFluor 780, ThermoFisher Scientific, 65-086514) and were centrifugated to obtain cell pellets prior to western blot analysis.

Recruitment

These two tumors were obtained from breast cancer patients and selected for their epithelial-mesenchymal profiles (CD44high).

Ethics oversight

CRCM Paoli Calmette

Note that full information on the approval of the study protocol must also be provided in the manuscript.

\section{ChIP-seq}

\section{Data deposition}

$\bigotimes$ Confirm that both raw and final processed data have been deposited in a public database such as GEO.

$\bigotimes$ Confirm that you have deposited or provided access to graph files (e.g. BED files) for the called peaks.

\section{Data access links}

May remain private before publication.

Files in database submission

Genome browser session (e.g. UCSC)
Code employed for ChIP-seq and RNA-seq data analyses are available on Github at https://github.com/nservant/EMTiron.

ChIP-Seq read counts of H3K9me2, H3K9me3, H3K4me3, H3K27me3 and RNA-seq

https://genome.ucsc.edu/cgi-bin/hgTracks?

hgS_doOtherUser=submit\&hgS_otherUserName=Nservant\&hgS_otherUserSessionName=H3K9me2_EMTIron 
Methodology

Replicates

Sequencing depth

Peak calling parameters

Data quality

Software
ChIP-Seq was performed in three independent biological replicates for $\mathrm{H} 3 \mathrm{~K} 9 \mathrm{me} 2$ and two independent biological replicates for the other marks.

Single End 50 base pairs

Total number of reads

$>$ mean(c(49842490, 35563313, 32423816, 41089015, 35739274, 26786819))

[1] 36907454

Number of uniquely aligned reads

$>$ mean(c(41810318, 29759212, 27040802, 33664856, 29995477, 22647165))

[1] 30819638

H3K4me3 (Diagenode, C15410003-50), H3K9me2 (Cell Signaling, 4658S, WB: 1:1000), H3K9me3 (Cell Signaling, 13969S), H3K27me3 (Cell Signaling, 9733S)

H3K9me2 is a repressive histone mark covering extremely broad domains. Peak calling methods are not suitable to identify these large domains. For the purpose of this study, we did not use peak information, but quantified instead ChIP enrichment at the level of genes.

Three replicates of each conditions have been performed as well as a control input for each condition.

$\mathrm{H} 3 \mathrm{~K} 9 \mathrm{me} 2$ signal is known to cover broad domains. We validated the profiles of our H3K9me2 samples using the EPIC domain caller and defining large repressive domains (LOCKs), as already proposed by MC Donald et al. 2018.

Raw sequencing reads were aligned with the Bowtie2 software on the Drosophila Melanogaster reference genome to extract reads coming from spike-in chromatin and on the Human hg38 reference genome.

PCR duplicates were removed from aligned data using Picard tools, and genome-browser tracks files were generated using the Deeptools suite. Counts of aligned reads per human gene have been generated using the FeatureCounts software and the gene coordinates extracted from the GENCODE annotation file. Differential analysis has been performed at the gene level with the limma package. Custom scripts are available at https://github.com/nservant/EMTiron.

\section{Flow Cytometry}

\section{Plots}

Confirm that:

\The axis labels state the marker and fluorochrome used (e.g. CD4-FITC).

ХThe axis scales are clearly visible. Include numbers along axes only for bottom left plot of group (a 'group' is an analysis of identical markers).

$\bigotimes$ All plots are contour plots with outliers or pseudocolor plots.

$\bigotimes$ A numerical value for number of cells or percentage (with statistics) is provided.

\section{Methodology}

Sample preparation

Instrument

Software

Cell population abundance

Gating strategy

$\bigotimes$ Tick this box to confirm that a figure exemplifying the gating strategy is provided in the Supplementary Information.

BD Accuri C6 (BD Biosciences)

\begin{abstract}
Fluorescent probes were used as described in the Reagents section. For surface staining with antibodies, cells were suspended in ice-cold $1 \times$ PBS containing $2 \%$ FBS and $1 \mathrm{mM}$ EDTA (incubation buffer) and were incubated for 20 min at $4{ }^{\circ} \mathrm{C}$ with the relevant antibody. Cells were then washed twice with ice-cold $1 \times$ PBS and suspended in incubation buffer prior to being analyzed by flow cytometry. For each condition, at least 10000 cells were counted. Data were recorded on a BD Accuri C6 (BD Biosciences) and processed using Cell Quest (BD Biosciences) and FlowJo (FLOWJO, LLC).
\end{abstract}

Data were processed using Cell Quest (BD Biosciences) and FlowJo (FLOWJO, LLC) software.

Only live cells were analysed as determined by the FSC/SCC gating.

FSC/SCC gates were used to select the bulk of the live cells, which was typically $>90 \%$. To determine fluorescence positive and negative cells, FSC-A was plotted against the relevant fluorescent channel, and converted into a histogram where appropriate. 